\title{
MULTISPECIES TRANSPORT THEORY FOR AXISYMMETRIC ROTATING PLASMAS
}

BY

M. TESSAROTTO AND R.B. WHITE

January 1992

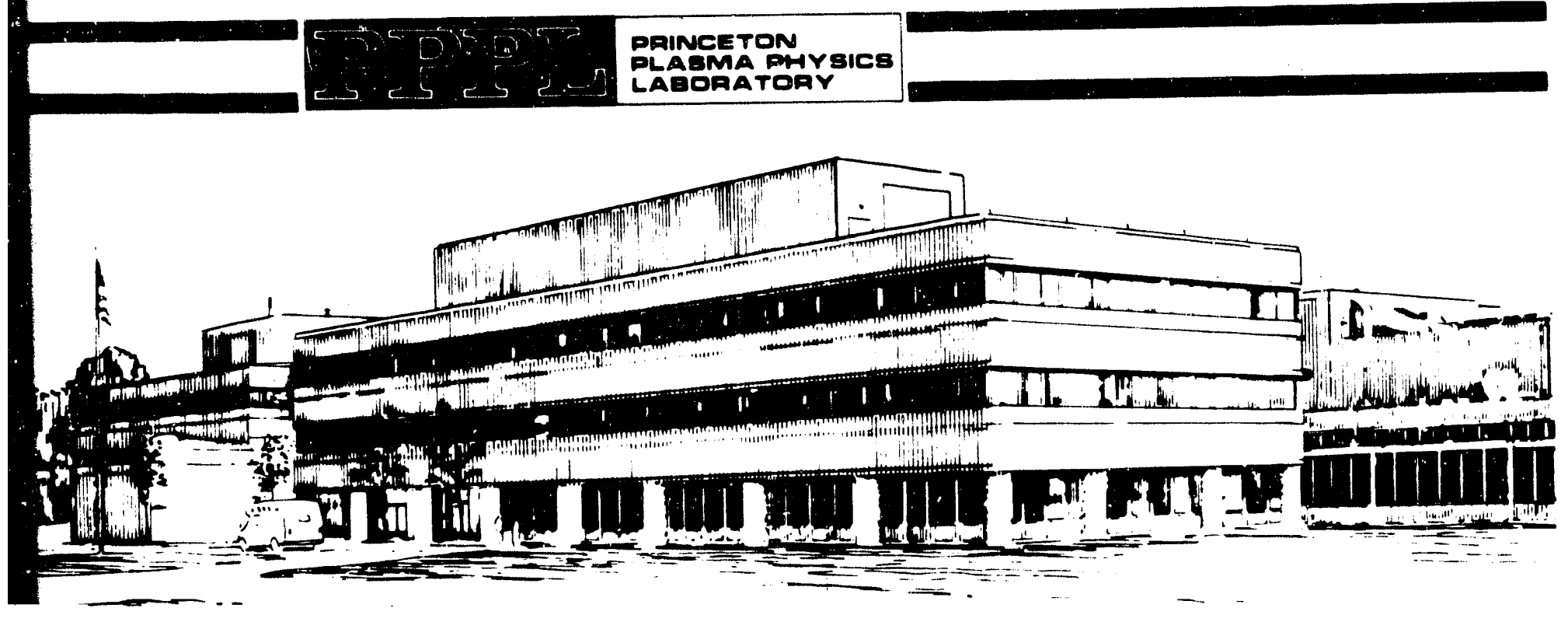




\section{NOTICE}

This report was prepared as an account of work sponsored by an agency of the United States Government. Neither the United States Government nor any agency thereof, nor any of their employees, makes any warranty, express or implied, or assumes any legal liability or responsibility for the accuracy, completeness, or usefulness of any information, apparatus, product, or process disclosed, or represents that its use would not infringe privately owned rights. Reference herein to any specific commercial produce, process, or service by trade name, trademark, manufacturer, or otherwise, does not necessarily constitute or imply its endorsement, recommendation, or favoring by the United States Government or any agency thereof. The views and opinions of authors expressed herein do not necessarily state or reflect those of the United States Government or any agency thereof.

\section{NOTICE}

This report has been reproduced directly from the best available copy.

Available to DOE and DOE contractors from the:

Office of Scientific and Technical Information

P.O. Box 62

Oak Ridge, TN 37831 ;

Prices available from (615) 576-8401.

Available to the public from the:

National Technical Information Service

U.S. Department of Commerce

5285 Port Royal Road

Springfield, Virginia 22161

703-487-4650 


\title{
MULTISPECIES TRANSPORT THEORY FOR AXISYMMETRIC ROTATING PLASMAS
}

\author{
Massimo Tessarotto \\ Dipartimento di Scienze Matematiche \\ Università di Trieste, TRIESTE, Italy \\ and \\ Roscoe B.White \\ Princeton Plasina Physics Laboratory \\ Princeton University, PRINCETON, N.J., USA
}

\section{ABSTRACT}

A reduced gyrolinetic equation is derived for a multi-species toroidal axisymmetric plasma with arbitrary toroidal differential rotation speeds and in the presence of a finite induced electric field.

The kinetic equation obtained, extending previous results obtained by Hinton and Wong [1985] and by Catto, Bernstein and Tessarotto [1987,1988], has a form suited for transport applications, via variational techniques; in particular it exhibits the feature that all source terms, including the Spitzer source term, carrying the contribution due to the inductive electric field, appear to be acted upon by the collision operator. Moreover, the equation displays a new contribution due to "explicit" velocity perturbations, here proven to be consistent with transport ordering, whose evaluation appears relevant for transport calculations.

In addition, general expressions are obtained for the neoclassical fluxes in terms of a variational principie, as well as for the classical ones, retaining, in both cases, the contributions due to Spitzer's inductive terms. 


\section{I - INTRODUCTION}

Until the theoretical work of Hinton and Wong [1] and Catto, Bernstein and Tessarotto [2,3], investigations of collisional transport in axisymmetric toroidal magnetoplasmas (i.e., Tokamaks), were limited to small toroidal rotation speeds and small toroidal differential rotation (i.e., small shear in the toroidal rotation speeds) [5-10].

The work of Catto et al. [2] extended the formulation of Hinton and Wong [1] , allowing more general expressions for the transport coefficients to be obtained and yielding, as well, a simpler derivation for the reduced gyrokinetic equation which advances in time the one particle kinetic distribution function.

The purpose of this paper is to carry out a systematic generalization of the approach previously developed in Reference [2] as appropriate for a multi-species plasma. In doing this the inductive Spitzer source term shall be consistently retained, by making use of a subsidiary multispecies Spitzer problem.

We consider a class of rotating equilibrium states, which allow finite velocity perturbations and related shear effects (differential toroidal rotation) due to the radial dependence of the mean angular velocity carried by the center of mass of the fluid element; moreover, in analogy to a previous work [3], we introduce a representation for the equilibrium states, which allows the introduction of explicit velocity perturbations produced by nonvanishing relative toroidal rotation speeds among the particle species.

The evolution of such general equilibrium states is investigated in the framework of a transport theory, i.e., all relevant dynamical variables are required to obey a transport ordering appropriate to strongly rotating magnetoplasmas $[1,2]$.

To this end, the gyrokinetic equation, which advances in time the first-order perturbation of the kinetic distribution function, is derived in a "reduced" form, in the sense that, without luss of generality or restrictions on the validity of the gyrokinetic equation, all source terms, including the so-called Spizer source term, appear inside the collision term, i.e., are given in terms of explicit corrections of the equilibrium distribution function.

Both the form of the reduced kinetic equation and the representation used for the 
equilibrium states are features potentially useful when using variational techniques for transport theory investigations of multispecies plasmas.

The derivation of the reduced kinetic equation is given, in Sec. 2, in terms of an asymptotic expansion technique based on a suitable choice of the equilibrium distribution function, chosen with the criterion of being the most general one consistent with the requirements of strong toroidal rotation and differential rotation speeds, as well as the existence of small but nonvanishing velocity perturbations due to relative angular velocities among the particle species as well as a finite inductive electric field.

The investigation of the constraints placed on the equilibrium kinetic distribution function, as well as on the equilibrium electromagnetic field and the inductive electric field, by such an ordering enables generalization of the customary gyrokinetic equation to account, in particular, for the aforementioned velocity perturbations.

It is found that the equilibrium distribution, as well as its first order perturbations, are consistent with Maxwell's equations, in particular fulfill not only quasi-neutrality and Ampere's law but also the related equation of hydromagnetic equilibrium, determining the equilibrium magnetic field. An analogous consistency is found with the Faraday law as well. These are important features, since it follows that, such velocity perturbations give, generally, a nonvanishing contribution to the toroidal current density.

In Sec. 2 a constrained variational formulation is given for the reduced gyrokinetic equation, extending in several ways the original variational approach due to Rosenbluth and coworkers [9]; in particular it allows a display of the simple relationship existing between the extremal value of the variational functional, the neoclassical fluxes, and the surface-average of entropy production rate.

In Sec. 3 an explicit solution is given for the auxiliary multi-species Spitzer problem. The approximate analytic solution obtained is, of course, useful for explicit evaluation of the inductive contributions to the transport matrix and the fluxes.

Finally, in Sec. 4, the constraints imposed by the transport ordering are investigated, the relevant transport equations are derived and, furthermore, explicit expressions are obtained, in terms of appropriate moment equations, for the classical fluxes. 


\section{2 - DERIVATION OF A REDUCED GYROKINETIC EQUATION AND ITS VARIATIONAL FORMUIATION}

In this Section the gyrokinetic kinetic equation will be obtained in a suitably "reduced" form, by employing a transport ordering, analogous to that used in Reference [2], and, in particular, apt for describing the collisional diffusion of a toroidal plasma in the presence of strong toroidal rotation and finite toroidal inductive electric field. The aim is to express the reduced kinetic equation in a form suitable for variational transport calculations in the case of multiple-species (say, $n$ species) plasmas.

The basic assumptions of the inodel are first briefly recalled for later convenience.

Thus we consider a toroidal axisymmetric magnetic configuration exhibiting nested magnetic surfaces, for which the magnetic field can be represented in the form:

$$
\underline{B}=\nabla \Psi \times \nabla v+I(\Psi, t) \nabla \vartheta=\underline{B}_{\mathrm{P}}+\underline{B}_{\mathrm{T}}
$$

[where, respectively, $\underline{\mathrm{B}}_{\mathrm{P}}=\mathrm{B}_{\mathrm{P}} \hat{\mathrm{e}}_{\mathrm{P}}=\nabla \Psi \times \nabla \vartheta, \underline{\mathrm{B}}_{\mathrm{T}}=\mathrm{B}_{\mathrm{T}} \hat{\mathrm{e}}_{\vartheta}=\mathrm{I}(\Psi, \mathrm{t}) \nabla \vartheta$ and $(\vartheta, \chi, \Psi)$ denote a set of toroidal magnetic flux coordinates] and require, for consistency with the previous representation that to lowest order in a Larmor radius expansion [i.e., an asymptotic expansion in terms of $\varepsilon_{\mathrm{s}} \sim \mathrm{O}\left(1 / \Omega_{\mathrm{s}}\right)$, with $\Omega_{\mathrm{s}}=\mathrm{Z}_{\mathrm{s}} \mathrm{e} / \mathrm{M}_{\mathrm{s}} \mathrm{cB}$ the Larmor frequency] there results:

$$
\underline{\mathrm{J}} . \nabla \Psi=0
$$

[here $\underline{\mathrm{J}}=\Sigma_{\mathrm{s}=1, \mathrm{n}} \mathrm{Z}_{\mathrm{s}} \mathrm{e} \underline{\mathrm{V}}_{\mathrm{s}}$ denotes the current density vector, whereas $\underline{\mathrm{V}}_{\mathrm{s}}=\frac{1}{\mathrm{~N}_{\mathrm{s}}} \int \mathrm{d}^{3} \mathrm{v} \underline{v}_{\mathrm{s}}(\underline{\mathrm{r}}, \underline{\mathrm{v}}, \mathrm{t})$ and $N_{s}=\int d^{3} v_{s}(\underline{r}, \underline{v}, t)$ are the mean flow velocity and number density carried by the s-th particle species]. The vector potential, in the Coulomb gauge ( $\nabla . \underline{A}=0$ ), corresponding to the previous representation of $\underline{B}$, reads:

$$
\underline{A}=\nabla g(\chi, \Psi, t) \times \nabla \vartheta+\Psi \nabla \vartheta
$$

where $g$ is related to $I(\Psi, t)$ through the differential equation:

$$
\nabla \cdot\left(\mathrm{R}^{-2} \nabla \mathrm{g}\right)=-\mathrm{RI}(\Psi, \mathrm{t}) \text {. }
$$

Hence the electric field can be represented as the sum of a divergenge-free and a curl 
free parts as:

$$
\underline{E}=-\nabla \Phi+\nabla G x \nabla \vartheta+H \nabla \vartheta
$$

with $\mathrm{G}=-c^{-1} \frac{\partial \mathrm{g}}{\partial \mathrm{t}}, \mathrm{H}=-\mathrm{c}^{-1} \frac{\partial \Psi}{\partial \mathrm{t}}$ and the toroidal component of the inductive electric field, hence, follows:

$$
(\mathrm{E}+\nabla \Phi) \cdot \hat{\mathrm{e}}_{\vartheta}=-\frac{1}{\mathrm{c}} \frac{\partial \Psi}{\partial \mathrm{t}}|\nabla \vartheta|=-\frac{1}{\mathrm{c} R} \frac{\partial \Psi}{\partial \mathrm{t}} .
$$

As in Reference [2], to treat large toroidal rotation as well as large toroidal differential rotation speeds, we shall require that, expanding the particle kinetic distribution function in the Larmor radius, its lowest order contribution, $f_{0, s}$, be a toroidally rotating local Maxwellian distribution. However, in analogy with Reference [3], we shall allow the particle species to have different rotation speeds, requiring at the same time, that the relative rotation speeds be small, in the Larmor radius ordering, with respect to the thermal speeds. To this purpose, let us introduce for thermal subdomain of velocity space ( defined by the condition that the kinetic energy be of the same order of the species temperature ) in which, the transport ordering, imposing for all physically relevant dynamical variabies ( here s denotes an arbitrary ionic species ):

$$
\begin{aligned}
& \frac{\frac{1}{\Omega_{\mathrm{s}}} \frac{\partial}{\partial t} \operatorname{lnf} \sim \frac{1}{\Omega_{\mathrm{s}}} \frac{\partial}{\partial t} \ln \Psi \sim \frac{1}{\Omega_{\mathrm{s}}} \frac{\partial}{\partial t} \ln \mathrm{B} \sim \frac{1}{\Omega_{\mathrm{s}}} \frac{\partial}{\partial t} \ln \mathrm{E} \sim \frac{1}{\Omega_{\mathrm{s}}} \frac{\partial}{\partial t} \ln \Phi \ldots \sim \mathrm{O}\left(\varepsilon_{\mathrm{s}}{ }^{3}\right)}{\frac{|\underline{\mathrm{E}}+\nabla \Phi|}{\mid \underline{\mathrm{E} \mid}} \sim \mathrm{O}\left(\varepsilon_{\mathrm{s}}{ }^{2}\right)} \\
& \frac{v_{\mathrm{eff}}}{\Omega_{\mathrm{s}}} \sim \frac{\omega}{\Omega_{\mathrm{s}}} \sim \frac{\Psi_{* \mathrm{~s}}-\Psi}{\Psi} \sim \mathrm{O}\left(\varepsilon_{\mathrm{s}}\right) \\
& \frac{\omega-\omega_{\mathrm{s}}}{\Omega_{\mathrm{s}}} \sim \mathrm{O}\left(\varepsilon_{\mathrm{s}}{ }^{2}\right) \\
& \frac{<\omega>-\omega_{\mathrm{s}}}{<\omega>} \sim \mathrm{O}\left(\varepsilon_{\mathrm{s}}\right)
\end{aligned}
$$

where $\Psi_{* \mathrm{~s}}$ is the exact invariant of motion $\Psi_{* \mathrm{~s}}=\Psi+\frac{\mathrm{M}_{\mathrm{s}} \mathrm{c}}{\mathrm{Z}_{\mathrm{s}} \mathrm{e}} \mathrm{Rv} \cdot \hat{\mathrm{e}}_{\vartheta}=\frac{\mathrm{c}}{\mathrm{Z}_{\mathrm{s}} \mathrm{e}} \mathrm{P}_{\vartheta}$ with $\mathrm{P}_{\vartheta}$ the canonical momentum conjugate to $\vartheta, v_{\mathrm{s}}^{\text {eff }}$ is an effective collision frequency and $\omega=\omega(\underline{r}, t), \omega_{s}=\omega_{s}(\underline{r}, t)$ are the toroidal angular velocity carried by the fluid element, 
respectively, of the center-of-mass and of the s-th species, i.e.:

$$
\omega=\frac{\Sigma_{s} M_{s} \int d^{3} v \underline{v} \cdot \hat{e}_{\vartheta}}{R \Sigma_{s} M_{s} N_{s}}=\frac{\Sigma_{s} M_{s} \int d^{3} v \underline{v} \cdot \hat{e}_{\vartheta} f_{s}}{R \Sigma_{s} M_{s} N_{s}}
$$

with

$$
\omega_{\mathrm{s}}=\frac{\int \mathrm{d}^{3} \mathrm{v} \underline{\mathrm{v}} \cdot \hat{\mathrm{e}} \mho_{\mathrm{s}}}{\mathrm{RN}_{\mathrm{s}}}
$$

It follows that $f_{0, s}$ can be chosen as a function of the exact as well as of the adiabatic invariants of particle motion. However, we are free to add to it arbitrary higher-order corrections in the Larmor radius; hence, it can always be chosen of the form of a drifting local Maxwellian distribution:

$$
f_{0, s}=\bar{N}_{s}\left(M_{s} / 2 \pi T T_{0, s}\right)^{3 / 2} \exp \left\{-M_{s}\left(\underline{v}-\underline{V}_{s}\right)^{2} / 2 T_{0, s}\right\}
$$

where, respectively, $\mathrm{T}_{0, s}=\mathrm{T}_{0, \mathrm{~s}}(\Psi, \mathrm{t})$ and $\underline{\mathrm{V}}_{\mathrm{s}}=<\omega_{\mathrm{s}}>\operatorname{Re} \hat{\mathrm{e}}_{\vartheta}$ are the temperature and the average toroidal rotation speed carried by the s-th particle species, where $\left\langle\omega_{\mathbf{s}}\right\rangle$ is the surface-averaged angular velocity $\left\langle\omega_{s}\right\rangle$ [and the brackets $"<>$ " denote the surface average:

$$
<\mathrm{A}>\left(\equiv<\mathrm{A}>_{\chi}\right)=\left(\mathrm{V}^{\prime}\right)^{-1} \oint \mathrm{d} \chi \mathrm{A}(\chi, \Psi, \vartheta, \mathrm{t})\left(\underline{\mathrm{B}}_{\mathrm{P}} \cdot \nabla \chi\right)^{-1}
$$

and $V^{\prime}=\oint d \chi\left(\underline{B}_{P} \cdot \nabla \chi\right)^{-1}$. In view of the ordering Eq. (2.11), $\underline{V}_{s}$ is, of course, required to differ from the toroidal baricentric rotation speed $\underline{V}=\langle\omega\rangle \operatorname{Re}_{\vartheta}$ by terms of first order in $\mathrm{O}\left(\varepsilon_{\mathrm{s}}\right) ; \mathrm{f}_{0, \mathrm{~s}}$, similarly, differs from the local maxwellian:

$$
f_{M_{, s}}=\bar{N}_{s}\left(M_{s} / 2 \pi T T_{0, s}\right)^{3 / 2} \exp \left\{-M_{s}(\underline{v}-\underline{V})^{2} / 2 T_{0, s}\right\}
$$

by terms of order $O\left(\varepsilon_{\mathrm{s}}\right)$, since, letting $\omega_{1, \mathrm{~s}} \equiv\left\langle\omega_{\mathrm{s}}\right\rangle-\langle\omega\rangle$ be the average angular velocity relative to the baricenter,

$$
\mathrm{f}_{0, \mathrm{~s}}-\mathrm{f}_{\mathrm{M}, \mathrm{s}}=\omega_{1, \mathrm{~s}} \frac{\partial}{\partial \omega_{1, \mathrm{~s}}} \mathrm{f}_{\mathrm{M}_{, \mathrm{s}}}+\mathrm{O}\left(\varepsilon_{\mathrm{s}}{ }^{2}\right)=\omega_{1, \mathrm{~s}} \operatorname{Re} \hat{e}_{\vartheta}(\underline{\mathrm{v}}-\underline{\mathrm{V}})_{\mathrm{T}_{0, \mathrm{~s}}}^{\mathrm{M}_{\mathrm{s}}} \mathrm{f}_{\mathrm{s}}+\mathrm{O}\left(\varepsilon_{\mathrm{s}}{ }^{2}\right)
$$


and $\omega_{1, s} \equiv\left\langle\omega_{\mathrm{s}}\right\rangle-\langle\omega\rangle$ represents the mean toroidal angular velocity relative to the center of mass.

To display the dependence in terms of the adiabatic and exact invariants of motion,

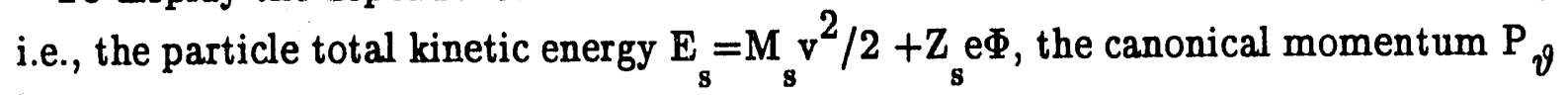
(or $\Psi_{* \mathrm{~s}}$ ) and the poloidal flux $\Psi$, let us introduce in analogy with Reference [2], the auxiliary dynamical variable $\mathrm{H}_{\mathrm{s}}\left(\left\langle\omega_{\mathrm{s}}\right\rangle\right)$ defined here as:

$$
\left.\left.\left.\mathrm{H}_{\mathrm{s}}\left(<\omega_{\mathrm{s}}\right\rangle\right)=\mathrm{E}_{\mathrm{s}}-\frac{\mathrm{Z}_{\mathrm{s}} \mathrm{e}}{\mathrm{c}}<\omega_{\mathrm{s}}\right\rangle\left(\Psi_{* \mathrm{~s}}-\Psi\right)-\frac{\mathrm{Z}_{\mathrm{s}} \mathrm{e}}{\mathrm{c}} \int_{0}^{\Psi} \mathrm{d} \Psi^{\prime}<\omega_{\mathrm{s}}\right\rangle
$$

[in the sequel, when unequivocal, we shall, instead, denote $\mathrm{H}_{\mathbf{s}}=\mathrm{H}_{\mathbf{s}}(\langle\omega\rangle)$ ] and related to the energy in the rotating frame $\mathrm{E}_{\mathrm{R}_{\mathrm{s}}}=\frac{1}{2} \mathrm{M}_{\mathrm{s}}\left(\underline{\mathrm{v}}-\underline{\mathrm{V}}_{\mathrm{s}}\right)^{2}+\mathrm{Z}_{\mathrm{s}} \mathrm{e} \Phi$ :

$$
\mathrm{H}_{\mathrm{s}}\left(<\omega_{\mathrm{s}}>\right)=\mathrm{E}_{\mathrm{R}_{\mathrm{s}}}-\frac{1}{2} \mathrm{M}_{\mathrm{s}} \mathrm{V}_{\mathrm{s}}^{2}-\frac{\mathrm{Z}_{\mathrm{s}} \mathrm{e}}{\mathrm{c}} \int_{0}^{\Psi} \mathrm{d}^{\prime} \Psi^{\prime}<\omega_{\mathrm{s}}>\text {. }
$$

Then it follows

$$
\frac{1}{2} M_{s}\left(\underline{v}-\underline{V}_{s}\right)^{2}=H_{s}\left(<\omega_{s}>\right)+\frac{1}{2} M_{s} V_{s}^{2}-Z_{s} e\left[\Phi-\frac{1}{c} \int_{0}^{\Psi} d \Psi^{\prime}<\omega_{s}>\right]
$$

and similarly

$$
\frac{1}{2} \mathrm{M}_{\mathrm{s}}(\underline{\mathrm{v}}-\underline{\mathrm{V}})^{2}=\mathrm{H}_{\mathrm{s}}(\langle\omega\rangle)+\frac{1}{2} \mathrm{M}_{\mathrm{s}} \mathrm{V}^{2}-\mathrm{Z}_{\mathrm{s}} \mathrm{e}\left[\Phi-\frac{1}{\mathrm{c}} \int_{0}^{\Psi} \mathrm{d} \Psi^{\prime}\langle\omega\rangle\right] .
$$

Represenling $\mathrm{f}_{\mathrm{M}, \mathrm{s}}$ in terms of $\mathrm{H}_{\mathrm{s}}(\langle\omega\rangle)$, there results:

$$
\begin{aligned}
\mathrm{f}_{\mathrm{M}_{, s}}=\mathrm{N}_{0, \mathrm{~s}}(\Psi, \mathrm{t}) \exp \left\{\frac{\mathrm{Z}_{\mathrm{s}} \mathrm{e}}{\mathrm{c} \mathrm{T}_{0, \mathrm{~s}}} \int_{0}^{\Psi} \mathrm{d} \Psi^{\prime} \omega_{1, \mathrm{~s}}\right\}\left(\mathrm{M}_{\mathrm{s}} / 2 \pi \mathrm{T}_{0, \mathrm{~s}}(\Psi, \mathrm{t})\right)^{3 / 2} \\
\exp \left\{-\mathrm{H}_{\mathrm{s}}(\langle\omega\rangle) / \mathrm{T}_{0, \mathrm{~s}}(\Psi, \mathrm{t})\right\}
\end{aligned}
$$

where $N_{0, s}$ is a reduced number density related to the number density $\bar{N}_{s}$ carried by $\mathrm{f}_{\mathrm{M}, \mathrm{s}}$ [see Eq. (2.14)] in terms of the relationship $\bar{N}_{\mathrm{s}}=\mathrm{N}_{0, \mathrm{~s}} \eta_{\mathrm{s}}(\langle\omega\rangle)$, with $\eta_{\mathrm{s}}(\langle\omega\rangle)$ the factor: 


$$
\eta_{s}(\langle\omega\rangle)=\exp \left\{-\left[\frac{1}{2} \mathrm{M}_{s} \mathrm{~V}^{2}-\mathrm{Z}_{\mathrm{s}} \mathrm{e}\left[\Phi-\frac{1}{\mathrm{c}} \int_{0}^{\Psi} \mathrm{d}^{\prime} \Psi^{\prime}\langle\omega\rangle\right]\right] / \mathrm{T}_{0, \mathrm{~s}}(\Psi, t)\right\}
$$

and $\mathrm{N}_{0,8}$ is required, for the existence of the equilibrium solution, to satisfy the constraint equation $\frac{\partial}{\partial \chi} N_{0, s}=0$; hence it follows that $N_{0, s}=N_{0, s}(\Psi, t)$. Of course, the drifting Maxwellian $f_{0, s}$ can similarly be represented in terms of in terms $H_{s}(\langle\omega\rangle)$, yielding:

$$
f_{0, s}=N_{0, s}(\Psi, t)\left(M_{s} / 2 \pi T_{0, s}(\Psi, t)\right)^{3 / 2} \exp \left\{-\bar{\omega}_{s}\left(<\omega_{s}>\right) / T_{0, s}(\Psi, t)\right\}
$$

To derive a gyrokinetic kinetic equation for the first order perturbation, we adopt here a technique analogous to that developed in Reference [2]. Hence, we define a function $f_{* s}$ suitably "close" to $f_{0, s}$, and hence to $f_{M, s}$, in the sense that:

$$
\frac{\mathrm{f}_{*_{\mathbf{s}}}-\mathrm{f}_{\mathrm{M}, \mathrm{s}}}{\mathrm{f}_{\mathrm{M}, \mathrm{s}}} \sim \mathrm{O}\left(\varepsilon_{\mathrm{s}}\right)
$$

and, of course, we impose the constraint, consistent with transport ordering,

$$
\frac{1}{\Omega_{s}} \frac{\partial}{\partial t} \ln f_{* s} \sim O\left(\varepsilon_{s}{ }^{3}\right)
$$

Introducing, therefore, the asymptotic Larmor-radius expansions:

$$
\begin{aligned}
& \mathrm{f}_{\mathrm{s}}=\mathrm{f}_{* \mathrm{~s}}+\mathrm{h}_{\mathrm{s}}=\mathrm{f}_{* \mathrm{~s}}+\varepsilon_{\mathrm{s}} \mathrm{h}_{1, \mathrm{~s}}+\varepsilon_{\mathrm{s}}^{2 h_{2, s}}+\ldots \\
& \mathrm{f}_{\mathrm{s}}=\mathrm{f}_{\mathrm{M}, \mathrm{s}}+\varepsilon_{\mathrm{s}} \mathrm{f}_{1, \mathrm{~s}}+\varepsilon_{\mathrm{s}} \mathrm{f}_{2, \mathrm{~s}}+\ldots
\end{aligned}
$$

it follows, in particular,

$$
f_{1, s}=h_{1, s}+f_{* s}-f_{M, s}
$$

where a suitable definition for $f_{* s}$ reads:

$$
f_{* s}=N_{* s}\left(M_{s} / 2 \pi T_{* s}\right)^{3 / 2} \exp \left\{-\left(H_{* s}+Q_{s}\right) / T_{* s}\right\} \text {. }
$$

As in Reference [2], $\mathrm{N}_{* \mathrm{~s}}$ and $\mathrm{T}_{* \mathrm{~s}}$ denote respectively the reduced number density $\mathrm{N}_{0, s}(\Psi, \mathrm{t})$ and temperature evaluated at $\Psi_{* s}$, i.e., $\mathrm{N}_{* \mathrm{~s}}=\mathrm{N}_{0, \mathrm{~s}}\left(\Psi_{* \mathrm{~s}}, \mathrm{t}\right), \mathrm{T}_{* \mathrm{~s}}=\mathrm{T}_{0, \mathrm{~s}}\left(\Psi_{* \mathrm{~s}}, \mathrm{t}\right)$, whereas $\mathrm{H}_{* \mathrm{~s}}$ is defined as: 


$$
H_{* s}=E_{s}-\frac{Z_{s} e}{C} \int_{0}^{\Psi_{* s}} d \Psi^{\prime}<\omega_{s}>.
$$

Finally, the dynamical variable $Q_{s}$, to be later defined, for consistency with Eq. (2.7) and Eq. (2.25), will be required to fulfill the orderings $\frac{Q_{\mathrm{s}}}{\mathrm{T}_{\mathrm{O}, \mathrm{s}}} \sim O\left(\varepsilon_{\mathrm{s}}\right)$ and $\frac{1}{\Omega_{\mathrm{s}}} \frac{\partial}{\partial t} \ln \mathrm{Q}_{\mathrm{s}} \sim$ $O\left(\varepsilon_{\mathrm{s}}{ }^{3}\right)$.

In terms of the definition of $f_{* s}$, given above, the explicit contribution to the kinetic distribution function, i.e., $f_{* s}-f_{M}$ can be evaluated. Thus, by Taylor expansion in terms of $\Psi_{* \mathrm{~s}}$ [invoking the ordering Eq. (2.9)], one obtains:

$$
\begin{aligned}
& H_{* s}=H_{s}(\langle\omega\rangle)-\frac{Z_{s} e}{c} \int_{0}^{\Psi} \Psi^{\prime} \omega_{1, s}-\frac{Z_{s} e^{2}}{c}\left(\Psi_{* s}-\Psi\right) \omega_{1, s}+ \\
& +\frac{\mathrm{Z}_{\mathrm{s}} \mathrm{e}}{2 \mathrm{C}}\left(\Psi_{* \mathrm{~s}}-\Psi\right)^{2} \frac{\partial}{\partial \Psi}<\omega_{\mathrm{s}}>
\end{aligned}
$$

and similarly letting $A=A\left(\Psi_{* s}, t\right)\left(A=N_{0, s}, T_{0, s}\right)$,

$$
\mathrm{A}_{* \mathrm{~s}}=\mathrm{A}(\Psi, \mathrm{t})+\left(\Psi_{* \mathrm{~s}}-\Psi\right) \frac{\partial}{\partial \Psi^{\mathrm{A}}} \mathrm{A}(\Psi, \mathrm{t})+\mathrm{O}\left(\varepsilon_{\mathrm{s}}{ }^{2}\right)
$$

Therefore, Taylor expanding $f_{* s}$ gives:

$$
\begin{aligned}
& \mathrm{f}_{* \mathrm{~s}}-\mathrm{f}_{\mathrm{M}_{, \mathrm{s}}}=\left(\Psi_{* \mathrm{~s}}-\Psi\right)\left[\frac{\partial}{\partial \Psi} \ln \mathrm{N}_{0, \mathrm{~s}}+\frac{\partial}{\partial \Psi} \ln \mathrm{T}_{0, \mathrm{~s}}\left[\frac{\mathrm{H}_{\mathrm{s}}}{\mathrm{T}_{0, \mathrm{~s}}}-\frac{3}{2}\right]\right] \mathrm{f}_{\mathrm{M}, \mathrm{s}}+
\end{aligned}
$$

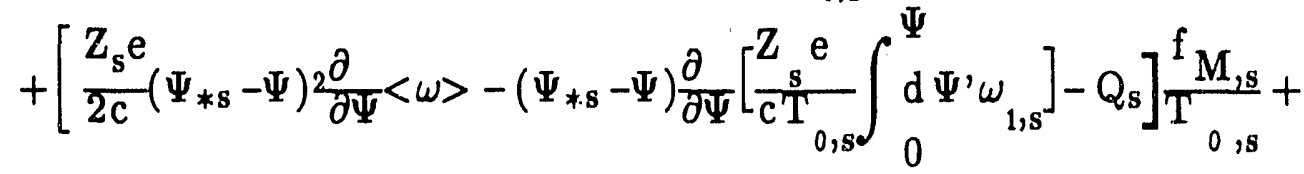

$$
\begin{aligned}
& +O\left(\varepsilon_{s}^{2}\right) .
\end{aligned}
$$

Hence, introducing the generalized forces

$$
\begin{aligned}
& \mathrm{A}_{1, \mathrm{~s}}=\frac{\partial}{\partial \Psi} \ln \mathrm{N}_{0, \mathrm{~s}}-\frac{3}{2} \frac{\partial}{\partial \Psi \ln \mathrm{T}_{0, \mathrm{~s}}} \\
& \mathrm{~A}_{2, \mathrm{~s}}=\frac{1}{\mathrm{~T}_{0, \mathrm{~s}}} \frac{\partial}{\partial \Psi^{2}} \ln \mathrm{T}_{0, \mathrm{~s}} \\
& \mathrm{~A}_{3, \mathrm{~s}}=\frac{1}{\mathrm{~T}_{0, \mathrm{~s}}} \frac{\partial}{\partial \Psi}<\omega>
\end{aligned}
$$




$$
\mathrm{A}_{4, \mathrm{~s}}=\frac{\partial}{\partial \Psi} \ln \mathrm{T}_{0, \mathrm{~s}} \frac{\mathrm{Z}_{\mathrm{s}} \mathrm{e}}{\mathrm{cT}_{0, \mathrm{~s}}} \int_{0}^{\Psi} \mathrm{d} \Psi^{\prime} \omega_{1, \mathrm{~s}}-\frac{\mathrm{Z}_{\mathrm{s}} \mathrm{e}}{\mathrm{cT} \mathrm{T}_{0, \mathrm{~s}} \omega_{1, \mathrm{~s}}}
$$

and the notations

$$
\begin{aligned}
& \mathrm{g}_{D_{s}}=\frac{M_{s} \mathrm{c}}{\mathrm{Z}_{\mathrm{s}} \mathrm{e}}\left(\mathrm{A}_{1, \mathrm{~s}}+\mathrm{A}_{4, \mathrm{~s}}+\mathrm{H}_{\mathrm{s}} \mathrm{A}_{2, \mathrm{~s}}\right) \operatorname{Re} \hat{\mathrm{e}}_{\vartheta} \mathrm{vf} \mathrm{M}_{, \mathrm{s}}+ \\
& +\frac{M_{s}^{2} c}{2 Z_{s} e^{2}} R\left(\underline{z} \cdot \hat{e}_{\vartheta}\right)^{2} A_{3, s} f_{M, s} \\
& \mathrm{~g}_{\mathrm{E}_{\mathrm{s}}}=-\mathrm{Q}_{\mathrm{s}} \mathrm{f}_{\mathrm{M}_{\mathrm{s}}} / \mathrm{T}_{0, \mathrm{~s}}
\end{aligned}
$$

where $\mathrm{g}_{\mathrm{D}_{\mathrm{s}}}$ shall be denoted as the collisionless diamagnetic contribution to the distribution function, there follows:

$$
f_{* s}=f_{M, s}+g_{D_{s}}+g_{E_{s}}+O\left(\varepsilon_{s}^{2}\right)
$$

We remark here (see also the discussion at the end of this Section) that in view of the equations defining the toroidal angular velocity $\omega_{s}$ [i.e., Eq. (2.13)], the generalized force $A_{4, s}$ is actually dependent on the remaining generalized forces.

In order to determine the kinetic equation advancing in time the first order perturbation $f_{1, s}$, we need to evaluate the total time derivative of $f_{* s}$. Thanks to conservation. of $\Psi_{* \mathrm{~s}}$, it reads:

$$
\frac{\mathrm{d}}{\mathrm{d} \mathrm{f}} \mathrm{f}_{* \mathrm{~s}}=-\frac{\dot{\mathrm{H}}_{*, \mathrm{~s}}+\dot{\mathrm{Q}}_{\mathrm{s}}}{\mathrm{T}_{* \mathrm{~s}}} \mathrm{f}_{* \mathrm{~s}}\left[1+\mathrm{O}\left(\varepsilon_{\mathrm{s}}\right)\right] \equiv \mathrm{S}_{\mathrm{s}}
$$

where, in order to express $S_{s}$, we Taylor expand in terms of $\Psi_{* s}$ all relevant dynamical variables, i.e., besides $f_{* \mathrm{~s}}$ [see Eq. (2.41)], $\mathrm{N}_{* \mathrm{~s}}, \mathrm{~T}_{* \mathrm{~s}}$ and $\mathrm{H}_{* \mathrm{~s}}$, appearing in the definition of $\mathrm{f}_{* \mathrm{~s}}$; hence there resuits $\mathrm{f}_{* \mathrm{~s}}-\mathrm{f}_{M, s}=O\left(\varepsilon_{\mathrm{s}}\right)$, whereas $\dot{\mathrm{H}}_{* \mathrm{~s}}$ reads: 


$$
\begin{gathered}
\dot{\mathrm{H}}_{* \mathrm{~s}}=\mathrm{Z}_{\mathrm{s}} \mathrm{e}\left[(\mathrm{E}+\nabla \Phi) \cdot \underline{\mathbf{v}}+\frac{\partial}{\partial t} \Phi\right]-\frac{\mathrm{Z}_{\mathrm{s}} \mathrm{e}}{\mathrm{c}} \int_{0}^{\mathrm{I}_{* \mathrm{~s}}} \Psi^{\prime} \frac{\partial}{\partial t}<\omega>\left.\right|_{\Psi}, \\
-\left.\frac{\mathrm{Z}_{\mathrm{s}} \mathrm{e}}{\mathrm{c}} \int_{0}^{\Psi_{* s}} \mathrm{~d} \Psi^{\prime} \frac{\partial}{\partial t} \omega_{1, \mathrm{~s}}\right|_{\Psi} ;
\end{gathered}
$$

Introducing, furthermore, the gyrophase

$$
\phi=\operatorname{arctg}\left\{\frac{(\underline{v}-\underline{V}) \cdot \hat{e}_{1}}{(\underline{v}-\underline{V}) \cdot \hat{e}_{2}}\right\}
$$

with $\left(\hat{\mathrm{e}}_{1}, \hat{\mathrm{e}}_{2}, \hat{\mathrm{e}}_{3}=\underline{\mathrm{b}}=\underline{\mathrm{B}} / \mathrm{B}\right)$ forming a triad of right-handed orthogonal unit vectors, the magnetic moment $\mu=\mathrm{w}^{2} / 2 \mathrm{~B}$ and using as velocity space variables $\left(\mathrm{H}_{\mathrm{s}}, \mu, \phi\right)$, the gyro-phase-average of a dynamical variable $A$ reads $\bar{A} \equiv<A\rangle_{\phi}=(1 / 2 \pi) \oint d \phi A\left(H_{s}, \mu, \phi, \underline{\mathbf{r}}, t\right)$, where we denote $A=\AA+\bar{A}$. Upon taking the gyrophase average of $\dot{H}_{* s}$ and $(d / d t) f_{* s}$, there follows:

$$
<\dot{\mathrm{H}}_{* \mathrm{~s}}>_{\phi} \stackrel{\sim}{=} \mathrm{Z}_{\mathrm{s}} \mathrm{e}\left[(\mathrm{E}+\nabla \Phi) \cdot \underline{\mathrm{b}} \mathrm{u}=-\mathrm{u} \frac{\mathrm{Z}_{\mathrm{s}} \mathrm{eB}_{\mathrm{T}}}{\mathrm{cR} \mathrm{B}} \frac{\partial}{\partial t} \Psi\right.
$$

and, furthermore

$$
\frac{\dot{\mathrm{H}}_{* \mathrm{~s}}-\left\langle\dot{\mathrm{H}}_{*_{\mathrm{s}}}\right\rangle_{\phi}}{\left.<\dot{\mathrm{H}}_{* \mathrm{~s}}\right\rangle_{\phi}} \sim \mathrm{O}\left(\varepsilon_{\mathrm{s}}^{0}\right)
$$

which imply:

$$
<\frac{\mathrm{d}}{\mathrm{d} t} \mathrm{f}_{* \mathrm{~s}}>_{\phi}=-<\mathrm{S}_{\mathrm{s}}>_{\phi}=\frac{1}{\mathrm{~T}_{0, \mathrm{~s}}}\left[\mathrm{u} \frac{\mathrm{Z}_{\mathrm{s}} \mathrm{eB} T \partial}{\mathrm{cRB} \partial \mathrm{t}} \Psi-<\dot{\mathrm{Q}}_{\mathrm{s}}>_{\phi}\right] \mathrm{f}_{\mathrm{M}_{, s}}\left[1+\mathrm{O}\left(\varepsilon_{\mathrm{s}}\right)\right] .
$$

The Fokker-Planck equation then reads in terms of $h_{s}$ :

$$
\frac{d}{d t} h_{s}-C_{s}(f \mid f)=S_{s}
$$

Neglecting contribution of second order in $\varepsilon_{\mathbf{s}}$, i.e., letting in particular $h_{\mathbf{s}}=$ $\mathrm{h}_{1, \mathrm{~s}}\left[1+\mathrm{O}\left(\varepsilon_{\mathrm{s}}\right)\right]$, the Fokker-Planck collision operator reads:

$$
C_{s}(f \mid f) \cong C_{s}\left(f_{M} \mid f-f_{M}\right)=C_{s}\left(f_{M} \mid h_{1}+f_{*}-f_{M}\right)
$$

where the term $f_{*}-f_{M}$ acts as a source term inside the collision operator. 
By taking the gyrophase average of Eq. (2.47), one obtains to leading order in $\varepsilon_{\mathrm{s}}$ :

$$
<\frac{\mathrm{d}}{\mathrm{dt}} \mathrm{h}_{1, \mathrm{~s}}>_{\phi}-\mathrm{C}_{\mathrm{s}}\left(\mathrm{f}_{\mathrm{M}} \mid<\mathrm{h}_{1}+>_{\phi}+\overline{\mathrm{g}}_{\mathrm{D}}-\overline{\mathrm{g}}_{\mathrm{E}}\right)=-\left\langle\mathrm{S}_{\mathrm{s}}\right\rangle_{\phi}
$$

where the source term $\left\langle\mathrm{S}_{q}\right\rangle_{\phi}$ can be written as the sum $\left\langle\mathrm{S}_{\mathrm{s}}\right\rangle_{\phi}=\mathrm{S}_{1 \mathrm{~s}}+\mathrm{S}_{2 \mathrm{~s}}$, with:

$$
\begin{aligned}
& \mathrm{S}_{\mathrm{is}}=-\mathrm{u} \frac{\mathrm{B}_{\mathrm{T}} \mathrm{R}}{\mathrm{B}} \frac{\mathrm{Z}_{\mathrm{s}} \mathrm{e}}{\mathrm{cT} \mathrm{T}_{0, \mathrm{~s}}}\left[\frac{\partial}{\partial t} \Psi \frac{1}{\mathrm{R}^{2}}-\left\langle\frac{\partial}{\partial t} \Psi \frac{1}{\mathrm{R}^{2}}\right)\right] \mathrm{f}_{\mathrm{M}_{, s}} \\
& \mathrm{~S}_{2 \mathrm{~s}}=-\left[\mathrm{u} \frac{\mathrm{B}_{\mathrm{T}} \mathrm{R}}{\mathrm{B}} \frac{\mathrm{Z}_{\mathrm{s}} \mathrm{e}}{\mathrm{cT} \mathrm{T}_{0, \mathrm{~s}}}\left\langle\frac{\partial}{\partial t} \Psi \frac{1}{\mathrm{R}^{2}}\right\rangle-\left\langle\dot{\mathrm{Q}}_{\mathrm{s}}\right\rangle_{\phi}\right] \mathrm{f}_{\mathrm{M}_{, s} .}
\end{aligned}
$$

Thus denoting:

$$
\mathrm{g}_{\chi^{\mathrm{s}}}=-\mathrm{B}_{\mathrm{T}^{\mathrm{R}}} \frac{\mathrm{Z}_{\mathrm{s}} \mathrm{e}}{\mathrm{c} \mathrm{T}_{0, \mathrm{~s}}} \mathrm{f}_{\mathrm{M}, \mathrm{s}} \int^{\chi} \mathrm{d} \chi^{\prime} \frac{1}{\mathrm{~B}_{\mathrm{P}}|\nabla \chi|}\left[\frac{1}{\mathrm{R}^{2}} \frac{\partial}{\partial \mathrm{t}} \Psi-\left\langle\frac{1 \partial}{\mathrm{R}^{2} \partial \mathrm{t}} \Psi\right\rangle\right]
$$

and letting

$$
\mathrm{h}_{1, \mathrm{~s}}=\mathrm{g}_{1, \mathrm{~s}}+\mathrm{g}_{\chi \mathrm{s}}
$$

since $\mathrm{g}_{\chi^{\mathrm{s}}}$ contributes nothing to the collision operator, Eq. (2.49) can be proven to yield [see also discussion below, from Eqs. (2.66) to (2.76)]:

$$
<\frac{\mathrm{d}}{\mathrm{d} t} \mathrm{~g}_{1, \mathrm{~s}}>_{\phi}-\mathrm{C}_{\mathrm{s}}\left(\mathrm{f}_{\mathrm{M}} \mid<\mathrm{h}_{1}+>_{\phi}+\overline{\mathrm{g}}_{\mathrm{D}}+\overline{\mathrm{g}}_{\mathrm{E}}\right)=-\mathrm{S}_{2 \mathrm{~s}} \text {. }
$$

Finally, to deal with the remaining source term, due to the inductive electric field, i.e., $S_{2 s}$, let us define the dynamical variable $Q_{s}$ as:

$$
\mathrm{Q}_{\mathrm{s}}=\frac{1}{M_{s}}\left[\frac{\mathrm{Z}_{\mathrm{s}} \mathrm{e}}{\mathrm{c}}\right]^{2} \mathrm{G}_{\mathrm{s}}\left(\mathrm{H}_{\mathrm{s}}\right) \int_{\Psi}^{\Psi_{*} \Psi_{\mathrm{s}}} \cdot\left\langle\frac{1 \partial}{\mathrm{R}^{2} \partial \mathrm{t}} \Psi\right\rangle_{\left(\Psi^{\prime}, t\right)}
$$

where $G_{s}\left(H_{s}\right)$ i.s the solution of the auxiliary stationary (Spitzer) problem:

$$
\mathrm{C}_{\mathrm{s}}\left(\mathrm{f}_{\mathrm{M}} \mid \mathrm{u} \frac{\mathrm{Z}}{\mathrm{T}_{0}} \mathrm{G}(\mathrm{H}) \mathrm{f}_{\mathrm{M}}\right)=-\mathrm{u} \frac{\mathrm{Z}_{\mathrm{s}}}{\mathrm{T}_{0, \mathrm{~s}}} \mathrm{f}_{M, s} \text {. }
$$

It is then immediate to verify that, consistently with the transport ordering, there results $Q_{s} / T_{0, s} \sim O\left(\varepsilon_{s}\right)$, and moreover:

$$
\begin{aligned}
& \frac{1}{\Omega_{\mathrm{s}}} \frac{1}{\mathrm{~T}_{0, \mathrm{~s}}}<\dot{\mathrm{Q}}_{\mathrm{s}}>_{\phi} \sim \mathrm{O}\left(\varepsilon_{\mathrm{s}}{ }^{3}\right) \\
& \frac{1}{\Omega_{\mathrm{s}}} \frac{1}{\mathrm{~T}_{0, \mathrm{~s}}}\left(\dot{\mathrm{Q}}_{\mathrm{s}}-<\dot{\mathrm{Q}}_{\mathrm{s}}>_{\phi}\right) \sim \mathrm{O}\left(\varepsilon_{\mathrm{s}}\right)
\end{aligned}
$$


where

$$
\dot{\mathrm{Q}}_{\mathrm{s}}-\left\langle\dot{\mathrm{Q}}_{\mathrm{s}}\right\rangle_{\phi}=-\mathrm{w} . \nabla \Psi \frac{1}{\mathrm{M}_{\mathrm{s}}}\left[\frac{\mathrm{Z}_{\mathrm{s}} \mathrm{e}}{\mathrm{c}}\right]^{2} \mathrm{G}_{\mathrm{s}}\left(\mathrm{H}_{\mathrm{s}}\right)\left\langle\frac{1 \partial}{\mathrm{R}^{2} \partial \mathrm{t}} \Psi\right\rangle\left[1+\mathrm{O}\left(\varepsilon_{\mathrm{s}}\right)\right] .
$$

Substituting in Eq. (2.54) the expression given by Eq. (2.55) for $Q_{S}$, Taylor expanded in $\Psi_{* s}$, to give

$$
\mathrm{g}_{\mathrm{E}_{\mathrm{s}}}=\left(\Psi_{* s}-\Psi\right) \frac{1}{\mathrm{M}_{\mathrm{s}} \mathrm{T}_{0, \mathrm{~s}}}\left[\frac{\mathrm{Z}_{\mathrm{s}} \mathrm{C}}{\mathrm{c}}\right]^{2} \mathrm{G}_{\mathrm{s}}\left(\mathrm{H}_{\mathrm{s}}\right)\left\langle\frac{1 \partial}{\mathrm{R}^{2}} \tilde{\mathrm{\partial t}} \Psi \mathrm{f}_{\mathrm{M}, \mathrm{s}}\right.
$$

and hence respectively

$$
\begin{aligned}
& \overline{\mathrm{g}}_{\mathrm{E}_{\mathrm{s}}}=\frac{\mathrm{Z}_{\mathrm{s}} \mathrm{e}}{\mathrm{c} \mathrm{T}_{0, \mathrm{~s}}}\left[\langle\omega\rangle \mathrm{R}^{2}+\mathrm{uR} \underline{b} . \hat{e}_{\vartheta}\right] \mathrm{G}_{\mathrm{s}}\left(\mathrm{H}_{\mathrm{s}}\right)\left\langle\frac{1 \partial}{\mathrm{R}^{2} \partial \mathrm{t}} \Psi\right\rangle \mathrm{f}_{\mathrm{M}, \mathrm{s}}
\end{aligned}
$$

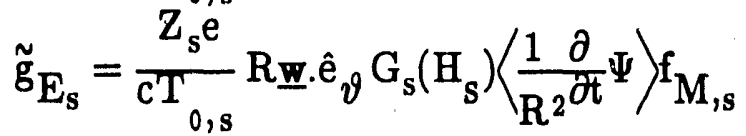

one finds from Eq. (2.55):

$$
\begin{gathered}
\mathrm{C}_{\mathrm{s}}\left(\mathrm{f}_{\mathrm{M}} \mid<\mathrm{h}_{1}>_{\phi}+\overline{\mathrm{g}}_{\mathrm{E}}\right)-\mathrm{u} \frac{\mathrm{B}_{\mathrm{T}}^{\mathrm{R}}}{\mathrm{B}} \frac{\mathrm{Z}_{\mathrm{s}} \mathrm{e}}{\mathrm{CT} \mathrm{T}_{0, s}}\left\langle\frac{i \partial}{\mathrm{R}^{2} \partial \mathrm{t}} \Psi\right)_{\mathrm{M}_{\mathrm{s}}}=\mathrm{C}_{\mathrm{s}}\left(\mathrm{f}_{\mathrm{M}} \mid<\mathrm{h}_{1}>_{\phi}+\overline{\mathrm{g}}_{\mathrm{EV}}\right) \\
{\left[1+\mathrm{O}\left(\varepsilon_{\mathrm{s}}\right)\right] .}
\end{gathered}
$$

In view of Eq. (2.61), it follows, therefore:

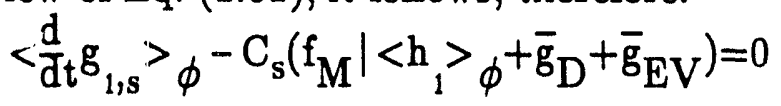

where:

$$
\mathrm{g}_{\mathrm{EV}}=-\frac{\mathrm{Z}_{\mathrm{s}} \mathrm{e}}{\mathrm{cT}}<\omega>\mathrm{R}^{2} \mathrm{G}_{\mathrm{s}, \mathrm{s}}\left(\mathrm{H}_{\mathrm{s}}\right)\left\langle\frac{1 \partial}{\mathrm{R}^{2} \partial \mathrm{t}} \Psi\right\rangle \mathrm{f}_{\mathrm{M}_{, \mathrm{s}}} .
$$

We finally need to elaborate on the term containing the gyrophase average of $(\mathrm{d} / \mathrm{dt}) \mathrm{g}_{1, \mathrm{~s}}$ in Eq. (2.64). Representing, hence, $\mathrm{g}_{1, \mathrm{~s}}$ as $\mathrm{g}_{1, \mathrm{~s}}=\overline{\mathrm{g}}_{1, \mathrm{~s}}+\tilde{\mathrm{g}}_{1, \mathrm{~s}}$, one obtair::

$$
{ }_{\mathrm{d} t}^{\mathrm{d}} \mathrm{g}_{1, \mathrm{~s}}=\left(\underline{\nabla} \nabla+\dot{\mathrm{H}}_{\mathrm{s}} \frac{\partial}{\partial \mathrm{H}_{\mathrm{s}}}+\dot{\mu}_{\frac{\partial}{\partial \mu}}+\dot{\phi} \frac{\partial}{\partial \phi}\right) \mathrm{g}_{1, \mathrm{~s}}
$$

which implies, from Eq. (2.47) that to leading order in $\varepsilon_{\mathrm{s}}, \tilde{\mathrm{g}}_{1, \mathrm{~s}}$ is determined by the equation:

$$
\left.-\Omega_{\mathrm{s}} \frac{\partial}{\partial \phi} \tilde{\mathrm{g}}_{1, \mathrm{~s}}=-\frac{1}{\mathrm{~T}_{0, \mathrm{~s}}}\left[\dot{\mathrm{Q}}_{\mathrm{s}}-<\dot{\mathrm{Q}}_{\mathrm{s}}\right\rangle_{\phi}\right] \mathrm{f}_{\mathrm{M}_{\mathrm{s}}}
$$

with solution: 


$$
\tilde{\mathrm{g}}_{1, \mathrm{~s}}=-\frac{\underline{\underline{w}} \underline{\underline{b}} \cdot \nabla \Psi}{\Omega_{\mathrm{s}}} \frac{1}{M_{\mathrm{s}} T_{0, s}}\left[\frac{\mathrm{Z}_{\mathrm{s}} \mathrm{e}}{\mathrm{c}}\right]^{2} \mathrm{G}_{\mathrm{s}}\left(\mathrm{H}_{\mathrm{s}}\right)\left\langle\frac{1 \partial}{\mathrm{R}^{2} \partial t} \Psi\right\rangle \mathrm{f}_{\mathrm{M}, \mathrm{s}} .
$$

Therefore, it follows that:

$$
<\frac{\mathrm{d}}{\mathrm{dt}}\left(\overline{\mathrm{g}}_{1, \mathbf{s}}+\tilde{\mathrm{g}}_{1, \mathrm{~s}}\right)>_{\phi}=\left(\mathbf{u} \underline{\mathrm{b}} \cdot \nabla \overline{\mathrm{g}}_{1, \mathrm{~s}}+<\underline{\underline{w}} \cdot \nabla \tilde{\mathrm{g}}_{1, \mathrm{~s}}>_{\phi}\right)\left[1+\mathrm{O}\left(\varepsilon_{\mathrm{s}}\right)\right]
$$

which, using the identity:

$$
\begin{aligned}
& <\Psi . \nabla\left[\frac{\underline{W} \underline{\underline{b}} \cdot \nabla \Psi}{\Omega_{s}}\right]>_{\phi}=\nabla_{D} \cdot \nabla \Psi \\
& \underline{\mathbf{v}}_{\mathrm{D}} \cdot \nabla \Psi=\mathrm{u} \frac{\mathbf{M}_{\mathrm{s}} \mathrm{c}}{\mathrm{Z}_{\mathrm{s}} \mathrm{e}} \underline{\mathbf{b}} \cdot \nabla\left[\left\langle\omega>\mathrm{R}^{2}+u R \hat{e}_{\vartheta} \underline{\mathbf{b}}\right]\right.
\end{aligned}
$$

implies:

$$
<\underline{w} \cdot \nabla \tilde{g}_{1, s}>_{\phi}=-\underline{\nabla}_{D} \cdot \nabla \Psi \frac{1}{M_{s} T}\left(\frac{Z_{s, s} \hat{e}}{c}\right]^{2} G_{s}\left(H_{s}\right)\left\langle\frac{1 \partial}{R^{2} \partial t} \Psi\right\rangle f_{M, s} .
$$

Denoting $\mathrm{g}_{\mathrm{DE}_{\mathrm{s}}}$ the solution of the differential equation:

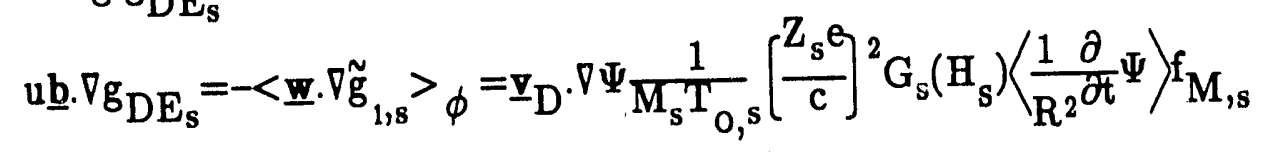

it follows:

$$
\mathrm{g}_{\mathrm{DE}_{\mathrm{s}}}=\frac{\mathrm{Z}_{\mathrm{s}} \mathrm{e}}{\mathrm{cT}}\left[\langle\omega\rangle \mathrm{R}^{2}+\mathrm{uRe} \dot{e}_{\vartheta} \underline{\mathrm{b}}\right] \mathrm{G}_{\mathrm{s}}\left(\mathrm{H}_{\mathrm{s}}\right)\left\langle\frac{1 \partial}{\mathrm{R}^{2} \partial \mathrm{t}} \Psi\right\rangle \mathrm{f}_{\mathrm{M}_{\mathrm{s}}}
$$

and hence letting

$$
\overline{\mathrm{g}}_{1, \mathbf{s}}=\mathbf{k}_{1, \mathbf{s}}+\mathrm{g}_{\mathrm{DE}_{\mathbf{s}}}
$$

there results

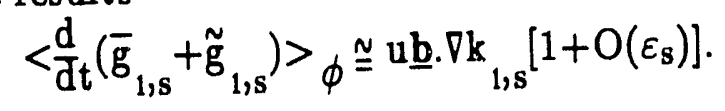

To complete the calculation, it is sufficient to introduce Eq. (2.76) in the first term of Eq. (2.64) and use the position Eq. (2.74) to give the seeked "reduced" form of the gyrokinetic equation, advancing in time the first order perturbation of the distribution function, i.e.,:

$$
\text { ub. } \nabla k_{1, s}-C_{s}\left(f_{M} \mid k_{1}+\bar{g}_{D}+G_{E}\right)=0 .
$$

Here $k_{1, s}$ is related to gyrophase-averaged first-order perturbation of the distribution function, $\mathrm{f}_{1, \mathrm{~s}}$, in terms terms of: 


$$
\mathrm{f}_{1, \mathrm{~s}}=\mathrm{h}_{1, \mathrm{~s}}+\left\langle\mathrm{f}_{* \mathrm{~s}}-\mathrm{f}_{\mathrm{M}, \mathrm{s}}^{\left({ }^{0}\right)}\right\rangle_{\phi}=\overline{\mathrm{g}}_{1, \mathrm{~s}}+\overline{\mathrm{g}}_{\mathrm{D}_{\mathrm{s}}}+\overline{\mathrm{g}}_{\mathrm{E}_{\mathrm{s}}}+\mathrm{g}_{\chi^{\mathrm{s}}}=\mathrm{k}{ }_{1, \mathrm{~s}}+\overline{\mathrm{g}}_{\mathrm{D}_{\mathrm{s}}}+\mathrm{G}_{\mathrm{E}_{\mathrm{s}}}+\mathrm{g}_{\chi^{\mathrm{s}}}
$$

and $G_{E_{\mathrm{s}}}$ denotes the inductive explicit term:

$$
G_{E_{s}}=u \frac{B_{T} R}{B} \frac{Z_{s} e}{C T=G_{s}}\left(H_{s}\right)\left\langle\frac{1 \partial}{R^{2} \partial t} \Psi\right\rangle f_{M, s}
$$

with $\mathrm{G}_{\mathrm{s}}\left(\mathrm{H}_{\mathrm{s}}\right)$ is the Spitzer's function. By introducing the thermodynamic force:

$$
A_{0, s}=\frac{Z_{s} e}{C T_{0, s}}\left\langle\frac{1 \partial}{R^{2} \partial^{2}} \Psi\right\rangle
$$

$\mathrm{G}_{\mathrm{E}_{\mathrm{s}}}$ reads:

$$
\mathrm{G}_{\mathrm{E}_{\mathrm{s}}}=\mathrm{u} \frac{\mathrm{B}_{\mathrm{T}}^{\mathrm{R}}}{\mathrm{B}} \mathrm{A}_{0, \mathrm{~s}} \mathrm{G}_{\mathrm{s}}\left(\mathrm{H}_{\mathrm{s}}\right) \mathrm{f}_{\mathrm{M}, \mathrm{s}} .
$$

Finally, in Eq. (2.77) there appears the gyrophase-average of the collisionless diamagnetic distribution, i.e., $\overline{\mathrm{g}}_{\mathrm{D}_{\mathrm{s}}}$, which from Eq. $(2.39)$ results to be:

$$
\begin{aligned}
& \overline{\mathrm{g}}_{\mathrm{D}_{\mathrm{s}}}=\frac{\mathrm{M}_{\mathrm{s}} \mathrm{c}}{\mathrm{Z}_{\mathrm{s}} \mathrm{e}}\left(\mathrm{A}_{1, \mathrm{~s}}+\mathrm{A}_{4, \mathrm{~s}}+\mathrm{H}_{\mathrm{s}} \mathrm{A}_{2, \mathrm{~s}}\right)\left[\langle\omega\rangle \mathrm{R}^{2}+\mathrm{uRe \hat { }} \vartheta \underline{\mathrm{b}}\right] \mathrm{f}_{\mathrm{M}, \mathrm{s}}+ \\
& +\frac{\mathrm{M}_{\mathrm{s}}^{2} \mathrm{c}}{2 \mathrm{Z}_{\mathrm{s}} \mathrm{e}^{2}} \mathrm{R}^{2}<\left(\mathrm{v} \cdot \hat{\mathrm{e}}_{\vartheta}\right)^{2}>_{\phi} \mathrm{A}_{3, \mathrm{~s}} \mathrm{f}_{\mathrm{M}, \mathrm{s}} \text {. }
\end{aligned}
$$

Similarly, the gyrophase-dependent part of the first order perturbation, i.e., $\mathfrak{f}_{1, \mathrm{~s}}$, reads:

$$
\dddot{\mathrm{I}}_{1, \mathrm{~s}}=\tilde{\mathrm{g}}_{1, \mathrm{~s}}+\tilde{\mathrm{g}}_{\mathrm{D}_{\mathrm{s}}}+\tilde{\mathrm{g}}_{\mathrm{E}_{\mathrm{s}}}
$$

and since to leading order $\tilde{\mathrm{g}}_{1, \mathrm{~s}}+\tilde{\mathrm{g}}_{\mathrm{E}_{\mathrm{s}}}=0$,

$$
\begin{aligned}
& \mathcal{H}_{1, \mathrm{~s}}=\tilde{\mathrm{g}}_{\mathrm{D}_{\mathrm{s}}}=\frac{\mathrm{M}_{\mathrm{s}} \mathrm{c}}{\mathrm{Z}_{\mathrm{s}} \mathrm{e}}\left(\mathrm{A}_{1, \mathrm{~s}}+\mathrm{A}_{4, \mathrm{~s}}+\mathrm{H}_{\mathrm{s}} \mathrm{A}_{2, \mathrm{~s}}\right) \operatorname{Re} \hat{e}_{\vartheta} \underline{\underline{w} \mathrm{w}_{\mathrm{M}, \mathrm{s}}}+ \\
& +\frac{M_{s}^{2} c}{2 Z_{s} e^{2}} R^{2}\left[\left(v \cdot \hat{e}_{\vartheta}\right)^{2}-<\left(v . \hat{e}_{\vartheta}\right)^{2}>_{\phi}\right] A_{3, s} f_{M, s} \text {. }
\end{aligned}
$$

The whole discussion, based on the introduction of the explicit corrections to the equilibrium distribution function carried by the dynamical variable $\mathrm{Q}_{\mathrm{S}}$ [see Eq. (2.55)], leads, therefore, to the conclusion that, the Spitzer source term can be exactly transformed in an explicit contribution of the distribution function $f$, without need of invoking additional assumptions concerning the plasma collisionality [9]; in addition we infer 
that, to leading order, $\mathfrak{f}_{1, \mathrm{~s}}$ is, actually, independent of the inductive electric Eieid.

A further significant result, useful to gain insight into the mechanisms of collisional transport in a rotating magnetoplasma, is the inclusion of self-consistent explicit velocity perturbations carried by the surface-averaged toroidal angular rotation speed (i.e., $\left\langle\omega_{\mathrm{s}}\right\rangle$ ). In the reduced gyrokinetic kinetic equativn here obtained [Eq. (2.77)], besides the customary driving forces related to spatial (radial) gradients of density, temperature $\left(A_{1, s}\right.$ and $\left.A_{2, s}\right)$ and toroidal angular velocity $\left(A_{3, s}\right)$, there appears the explicit dependence in terms of a suitable weighted average of the inductive electric field $\left(A_{0,5}\right)$ and the toroidal angular velocity relative to the center of mass $\left(A_{4, s}\right)$.

It is also to be noted that since $A_{4,8}$ is not independent of the remaining generalized forces, one can always define an effective force $A_{1, s}^{\prime}$ :

$$
\mathrm{A}_{1, \mathrm{~s}}^{\prime}=\frac{\partial}{\partial \Psi} \ln N_{0, \mathrm{~s}}-\frac{3}{2} \frac{\partial}{\partial \Psi^{2}} \ln \mathrm{T}_{0, \mathrm{~s}}=\mathrm{A}_{1, \mathrm{~s}}+\mathrm{A}_{4, \mathrm{~s}}
$$

in terms of the reduced density:

$$
N_{0, \mathrm{~s}}=\mathrm{N}_{0, \mathrm{~s}}(\Psi, \mathrm{t}) \exp \left\{\frac{\mathrm{Z}_{\mathrm{s}} \mathrm{e}}{\mathrm{cT}_{0, \mathrm{~s}}} \int_{0}^{\Psi} \mathrm{d} \Psi^{\prime} \omega_{1, \mathrm{~s}}\right\}
$$

thus formally recovering, in this respect, a formulation equivalent to that given previously by Hinton and Wong [1] and by Catto et al. [2].

However, in general, the explicit form given above in terms of $A_{4, s}$ seems relevant especially in view of transport applications, since it can be exploited for variational evaluations of transport coefficients.

Another important feature which appears particularly useful in view of variational calculations is the the fact that all source terms appearing in the reduced (gyrokinetic) equation appear through explicit contributions to the distribution function (i.e., $\overline{\mathrm{g}}_{\mathrm{D}_{\mathrm{s}}}$, $\mathrm{G}_{\mathrm{E}_{\mathrm{s}}}$ ) and act inside the collision operator.

The form of the reduced kinetic equation given represents, in several respects, a generalization of analogous equations obtained by Hinton and Wong [1] and by Catto et al. [2]: 1) it applies to a multi-component plasma; 2) it is particularly suitable for variational evaluation of transport coefficents, in particular related to the inductive 
electric field (Ware pinch and bootstrap current); 3 ) it is valid for arbitrary toroidal rotation speeds, finite toroidal differential rotation speeds and arbitrary plasma collisionalities.

That a variational formulation can actually be given for the reduced kinetic equation is well known in neoclassical theory [9] and has been extended also to strong rotation theories $[1,2]$. Here we shall depart from the variational approaches used in References [1,2] adopting, instead, a constrained variational formulation generalizing that due to Rosenbluth and coworkers [9], later extended by Tessarotto [10] to a multispecies plasma and arbitrary aspect ratios.

For definiteness, to reach a variational formulation of this type, let us consider, without loss of generality, the case of an equal temperature plasma [i.e., all species having locally the same temperature $\left.\mathrm{T}_{0, \mathrm{~s}}=\mathrm{T}_{\mathrm{o}}(\Psi, \mathrm{t})\right]$. Let us introduce the three functionals $W\left(k_{1} \mid k_{1}\right)$ and $Q_{i}\left(k_{1} \mid k_{1}\right)(i=1,2)$, respectively defined as:

$$
\begin{aligned}
& \mathrm{W}\left(\mathrm{k}_{1} \mid \mathrm{k}_{1}\right)=\left\langle\mathrm{k}_{1}+\overline{\mathrm{g}}_{\mathrm{D}}+\mathrm{G}_{\mathrm{E}}\right| \mathrm{C}\left(\mathrm{f}_{\mathrm{M}} \mid \mathrm{k}_{1}+\overline{\mathrm{g}}_{\mathrm{D}}+\mathrm{G}_{\mathrm{E}}\right)> \\
& \begin{array}{l}
\mathrm{Q}_{1}\left(\mathrm{k}_{1} \mid \mathrm{k}_{1}\right)=<\mathrm{k}_{1}^{(\text {even })} \mid\left[\mathrm{u} \underline{\mathbf{b}} \cdot \nabla \mathrm{k}_{1}-\mathrm{C}\left(\mathrm{f}_{\mathrm{M}} \mid \mathrm{k}_{1}+\overline{\mathrm{g}}_{\mathrm{D}}+\mathrm{G}_{\mathrm{E}}\right)\right]^{(\text {even })}> \\
\mathrm{Q}_{2}\left(\mathrm{k}_{1} \mid \mathrm{k}_{1}\right)=<\mathrm{k}_{1}^{(\text {odd })} \mid\left[\mathrm{u} \underline{\mathbf{b}} \cdot \mathrm{\nabla k} \mathrm{k}_{1}-\mathrm{C}\left(\mathrm{f}_{\mathrm{M}} \mid \mathrm{k}_{1}+\overline{\mathrm{g}}_{\mathrm{D}}+\mathrm{G}_{\mathrm{E}}\right)\right]^{(\text {odd })}>
\end{array}
\end{aligned}
$$

where $\mathbf{k}_{1, \mathbf{s}}^{\text {(odd) }}\left(\mathbf{k}_{1, \mathrm{~s}}^{\text {(even) }}\right)$ is the odd (even) part of $\mathbf{k}_{1, \mathrm{~s}}$ with respect to the parallel velocity $\mathrm{u}$ and $\langle\mathrm{A} \mid \mathrm{B}\rangle$ is the (well known) scalar product:

$$
<A|B\rangle=\Sigma_{s=1, n}\left\langle\int d^{3}{ }^{3} A_{s} B_{s} / f_{M}, s\right.
$$

of two integrable vector functions $A(\underline{\mathbf{r}}, \underline{\underline{v}}, \mathbf{t})=\left(\mathrm{A}_{1}, \ldots, \mathrm{A}_{\mathbf{n}}\right), \mathrm{B}(\underline{\mathbf{r}}, \mathbf{\underline { \mathbf { v } }}, \mathrm{t})=\left(\mathrm{B}_{1},, \mathrm{~B}_{\mathbf{n}}\right)$, with $\mathrm{n}$ denoting the number of particle species.

Then, a variational formulation for Eq. (2.77) is given by the Euler equation

$$
\delta_{\mathrm{S}} \mathrm{W}\left(\mathbf{k}_{1} \mid \mathbf{k}_{1}\right)=0
$$

where the variations are performed with respect $\mathrm{k}_{1, \mathrm{~s}}^{\prime}=\mathrm{k}_{1, \mathrm{~s}}+\frac{\mathrm{Z}_{\mathrm{s}} \mathrm{e}}{\mathrm{c}}\left[\mathrm{Re} \hat{\mathrm{e}}_{\vartheta} \mathbf{w}\right] \omega_{1, \mathrm{~s}} \mathrm{f}_{\mathrm{M}_{, s}} / \mathrm{T}_{0, \mathrm{~s}}$ and the (odd and even parts of the) extremal functions $k_{1, s}$ fulfill the previous constraints Eqs.(2.88) and (2.89), hence:

$$
\delta_{\mathrm{S}} \mathrm{Q}_{\mathrm{i}}\left(\mathrm{k}_{1} \mid \mathrm{k}_{1}\right)=0 \quad(\mathrm{i}=1,2) .
$$


Notice that, for example, $\delta_{\mathrm{s}} \mathrm{W}\left(\mathrm{k}_{1} \mid \mathrm{k}_{1}\right)=\left.\frac{\partial}{\partial \alpha_{\mathrm{s}}} \mathrm{W}\left(\mathrm{k}_{1}(\alpha) \mid \mathrm{k}_{1}(\alpha)\right)\right|_{\alpha=0}$ denotes the first order variation of the functional, $\mathrm{k}_{1, \mathrm{~s}}\left(\alpha_{\mathrm{s}}\right)=\mathrm{k}_{1, \mathrm{~s}}^{\prime}+\alpha_{\mathrm{s}} \delta \mathrm{k}_{1, \mathrm{~s}}$ the varied function, with $\alpha_{\mathrm{s}} \in[0,1]$, and $\delta \mathbf{k}_{1, \mathrm{~s}}$ the variation of $\mathbf{k}_{1, \mathrm{~s}}^{\prime}$.

In a similar fashion, a variational formulation can be obtained from the Euler equation [i.e., Eq. (2.91)] also for the surface-averaged gyrokinetic equation:

$$
\left\langle\frac{\mathrm{B}}{\mathrm{u}} \mathrm{C}_{\mathrm{s}}\left(\mathrm{f}_{\mathrm{M}} \mid \mathrm{k}_{1}+\overline{\mathrm{g}}_{\mathrm{D}}+\mathrm{G}_{\mathrm{E}}\right)\right\rangle=0
$$

by imposing that the variation $\delta \mathbf{k}_{1, \mathrm{~s}}$ be constant on a toroidal magnetic surface ( $\Psi=$ const.).

In terms of the the variational functional $\mathrm{W}\left(\mathrm{k}_{1} \mid \mathrm{k}_{1}\right)$, one obtains the neoclassical fluxes for a multi-species rotating plasma. To display the relationship with the fluxes, we notice that the extremal value of the functional $W\left(k_{1} \mid k_{1}\right)$ reads:

$$
\begin{aligned}
\mathrm{W}\left(\mathrm{k}_{1} \mid \mathrm{k}_{1}\right) & =<\overline{\mathrm{g}}_{\mathrm{D}}+\mathrm{G}_{\mathrm{E}} \mid \mathrm{C}\left(\mathrm{f}_{\mathrm{M}} \mid \mathrm{k}_{1}+\overline{\mathrm{g}}_{\mathrm{D}}+\mathrm{G}_{\mathrm{E}}\right)>= \\
= & <\overline{\mathrm{g}}_{\mathrm{D}}\left|\mathrm{C}\left(\mathrm{f}_{\mathrm{M}} \mid \mathrm{k}_{1}+\overline{\mathrm{g}}_{\mathrm{D}}+\mathrm{G}_{\mathrm{E}}\right)>+<\mathrm{k}_{1}+\overline{\mathrm{g}}_{\mathrm{D}}+\mathrm{G}_{\mathrm{E}}\right| \frac{\mathrm{Ze}}{\mathrm{T}}_{\mathrm{O}} \mathrm{ub} .(\underline{\mathrm{E}}+\nabla \Phi)>
\end{aligned}
$$

namely

$$
\begin{gathered}
\mathrm{W}\left(\mathrm{k}_{1} \mid \mathrm{k}_{\mathrm{l}}\right)=\mathrm{\Sigma}_{\mathrm{s}=1, \mathrm{n}}\left[\left(\mathrm{A}_{1, \mathrm{~s}}+\mathrm{A}_{4, \mathrm{~s}}\right) \Gamma_{1, \mathrm{~s}}^{(\mathrm{n})}+\mathrm{A}_{2, \mathrm{~s}} \Gamma_{\mathrm{H}, \mathrm{s}}^{(\mathrm{n})_{+}}\right. \\
\left.+\mathrm{A}_{3, \mathrm{~s}} \mathrm{~L}_{\mathrm{s}}^{(\mathrm{n})}-\left\langle\mathrm{J}_{\| \mathrm{s}} \cdot \mathbf{b} \cdot(\underline{\mathrm{E}}+\nabla \Phi) / \mathrm{T}_{0, \mathrm{~s}}\right\rangle\right]
\end{gathered}
$$

where $J_{\| s}=Z_{s}$ e $d^{3}$ vuf is the current density contribution carried by the species $s$ and $\Gamma_{1, s}^{(n)}, \Gamma_{H, s}^{(n)}$ and $L_{s}^{(n)}$ are respectively the neoclassical particle, energy and angular momentum fluxes, i.e., for $\mathrm{X}_{\mathrm{s}}=1, \mathrm{H}_{\mathrm{s}}$ :

$$
\Gamma_{X, s}^{(n)}=\frac{M_{s}^{c}}{Z_{s} e}\left\langle R \int d^{3}{ }^{3} X_{s}\left\langle\underline{r} \cdot \hat{e}_{\vartheta}\right\rangle_{\phi} C_{s}\left(f_{M} \mid k_{1}+\bar{g}_{D}+G_{E}\right)\right\rangle
$$

and

$$
\mathrm{L}_{\mathbf{s}}^{(\mathrm{n})}=\frac{\mathrm{M}^{2} \mathrm{c}}{2 \mathrm{Z}_{\mathbf{s}} \mathrm{e}}\left\langle\mathrm{R}^{2} \int \mathrm{d}^{3} \mathrm{v}<\left(\mathbf{z} \cdot \hat{\mathrm{e}}_{\vartheta}\right)^{2}{ }_{\phi} \mathrm{C}_{\mathrm{s}}\left(\mathrm{f}_{\mathrm{M}} \mid \mathrm{k}_{1}+\overline{\mathrm{g}}_{\mathrm{D}}+\mathrm{G}_{\mathrm{E}}\right)\right\rangle .
$$

It is relevant to mention that, in analogy with weak-rotation theory $[9,10]$, the 
extremal functional $W\left(k_{1} \mid k_{1}\right)$ is related to the surface-averaged local entropy production in terms of the generalized forces and the transport matrix (see corresponding discussion in Appendix B).

Finally, straightforward is the extension of the previous constrained variational formulation to the case of Maxwellian equilibria of different temperatures, i.e., in which locally $T_{0, s}(\Psi, t) \neq T_{0}(\Psi, t)$ for all species (s) which ha,ve, in a suitable sense, "disparate" masses with respect to the remaining species, i.e., either their masses are much larger or much smaller (see discussion in Appendix C). 


\section{3 - AUXILIARY SPITZER PROBLEM}

In order to evaluate explicitly the source term $G_{E}$ in Eq. (2.77), the Spitzer function $\mathrm{G}_{\mathrm{s}}\left(\mathrm{H}_{\mathrm{s}}\right)$, solution of Eq. (2.56), must be determined in the case of a multiple-species rotating plasma.

The problem is easily reduced to the solution of a set of linear integro-differential equation for $\mathrm{G}_{\mathrm{s}}\left(\mathrm{H}_{\mathrm{s}}\right)(\mathrm{s}=1, \mathrm{n})$. The linearized Fokker-Planck collision operator reads in fact (Appendix A):

$$
\mathrm{C}_{\mathrm{s}}\left(\mathrm{f}_{\mathrm{M}} \mid \mathrm{u} \mathrm{T}_{0}^{\mathrm{Z}} \mathrm{G}(\mathrm{H}) \mathrm{f}_{\mathrm{M}}\right)=\mathrm{uL} \mathrm{s}_{\mathrm{s}}\left\{\frac{\mathrm{Z}}{\mathrm{T}_{0}} \mathrm{G}(\mathrm{H}) \mathrm{f}_{\mathrm{M}}\right\}
$$

where $\mathrm{L}$ is the linear integro-differential operator:

$$
\left.\mathrm{L}_{\mathrm{s}}\{\mathrm{F}\}=\mathrm{L}_{\mathrm{1}, \mathrm{s}}\left\{\mathrm{F}_{\mathrm{s}}\right\}+\mathrm{\Sigma}_{\mathrm{k}=1, \mathrm{n}_{2, \mathrm{k}}} \mathrm{L}_{\mathrm{k}}\right\}
$$

with $F_{s}\left(x_{k}\right)=\frac{Z_{s}}{T_{0, s}} G_{s}\left(H_{s}\right) f_{M, s}$ and $x_{s}=v^{\prime} / v_{t h, s}, v_{t h, s}=\left(2 T_{0, s} / M_{s}\right)^{1 / 2}$. Denoting by $\mathrm{U}_{\mathbf{s}}^{(1)}, \mathrm{U}_{\mathbf{s}}^{(2)}$ suitable energy integrals, given in Appendix A, the operator $\mathrm{L}_{1, \mathrm{~s}}\{\mathrm{~F}\}$ seads:

$$
L_{1, s}\left\{F_{s}\right\}=-\frac{1}{2} \frac{U_{s}^{(1)}}{v^{\prime 2}} F_{s}+\frac{1}{v^{\prime} 3} \frac{\partial}{\partial \nu^{\prime}}\left[v^{\prime 2}\left\{U_{s}^{(1)}-U_{s}(2)\right\} \frac{\partial}{\partial v^{\prime}}\left(v^{\prime} F_{s}\right)\right]
$$

whereas the integral operator $L_{2, k}\left\{F_{k}\right\}$ can be evaluated exactly [11] and reads:

$$
\begin{aligned}
& L_{2, k}\left\{F_{k}\right\}=8 \pi q_{s k} \frac{M_{k}^{s}}{M_{k}} f_{M, s}\left(x_{s}\right)\left\{\frac{4}{5} \alpha_{k s}^{2}\left[x_{k}^{2} \int_{0}^{x_{k}^{k}} d_{k} F_{k}(x)+x_{k}^{-3} \int_{0}^{x_{k}} d x x^{6} F_{k}(x)\right]+\right. \\
& -\frac{2}{3}\left[\mathrm{x}_{\mathrm{k}}^{-3} \int_{0}^{\mathrm{x}} \mathrm{dx} \mathrm{xx}^{4} \mathrm{~F}_{\mathrm{k}}(\mathrm{x})+\int_{\mathrm{x}_{\mathrm{k}}}^{\infty} \mathrm{dxx} \mathrm{F}_{\mathrm{k}}(\mathrm{x})\right]+\frac{2}{3} \alpha_{\mathrm{ks}}^{2}\left(1-\alpha_{\mathrm{sk}}^{2}\right) \\
& \left.\left[-2 x_{k}^{-3} \int_{0}^{x_{k}} d x x^{4} F_{k}(x)+\int_{x_{k}}^{\infty} d x x F_{k}(x)\right]+F_{k}\left(x_{k}\right)\right\}
\end{aligned}
$$

with $\alpha_{\text {sk }}=v_{\text {th, }}^{2} / v_{\text {th, }}^{2}$. 
Equation (2.56) results then:

$$
\mathrm{L}_{\mathrm{s}}\left\{\mathrm{T}_{\mathrm{o}}^{\mathrm{Z}} \mathrm{G}(\mathrm{H}) \mathrm{f}_{\mathrm{M}}\right\}=-\frac{\mathrm{Z}_{\mathrm{s}}}{\mathrm{T}_{0, \mathrm{~s}}} \mathrm{f}_{\mathrm{M}, \mathrm{s}}
$$

and can, in principle be solved numerically.

For transport application, however, an approximate analytic solution based on an expansion in terms of a suitable basis of orthonormal polynomials, functions of $x_{s}$, is more expedite.

An approximate solution method of this type, based on an expansion in terms of Sonine polynomials (Legendre's modified functions of order $3 / 2$ ), i.e., the basis $\left\{\mathrm{L}_{\mathrm{j}}^{3 / 2}\left(\mathrm{x}_{\mathrm{s}}^{2}\right), \mathrm{j} \in \mathrm{N}\right\}$ was used by Hirshman [12] to derive a three-term polynomial approximation to the Spitzer function $\mathrm{G}_{\mathrm{s}}\left(\mathrm{H}_{\mathrm{s}}\right)$.

To derive an explicit approximate solution for a multi-specias plasma, in an equivalent by simpler way, one can formally proceed by introducing an auxiliary set of orthonormal polynomials $\left\{\mathrm{K}_{\mathrm{j}, \mathrm{s}}\left(\mathrm{x}_{\mathbf{s}}^{2}\right), \mathrm{j} \in \mathrm{N}\right\}$, constructed in terms of the aforementioned Sonine polynomials and defined in such a way to fulfill the orthonormality conditions:

$$
\begin{array}{r}
\int d^{3} v \frac{2 u}{v_{t h, s}} K_{j, s}\left(x_{s}^{2}\right)\left[\sum_{k=1, n} C_{s k}\left[f_{M, k} \mid \frac{2 u}{v_{t h, s}} K_{i, s}\left(x_{s}^{2}\right) f_{M, s}\right]+\right. \\
\left.C_{s s}\left[f_{M, s} \mid \frac{2 u}{v_{t h, s}} K_{i, s}\left(x_{s}^{2}\right) f_{M, s}\right]\right]=-\delta_{i j}
\end{array}
$$

and letting:

$$
\begin{aligned}
& \mathrm{K}_{0, \mathrm{~s}}\left(\mathrm{x}_{\mathbf{s}}^{2}\right)=\alpha_{0, \mathrm{~s}} \\
& \mathrm{~K}_{1, \mathrm{~s}}\left(\mathrm{x}_{\mathrm{s}}^{2}\right)=\alpha,\left[1+\mathrm{a}{ }_{11, \mathrm{~s}} \mathrm{~L}_{1}^{3 / 2}\left(\mathrm{x}_{\mathrm{s}}^{2}\right)\right] \\
& \left.\left.\mathrm{K}_{2, \mathrm{~s}}\left(\mathrm{x}_{\mathrm{s}}^{2}\right)=\alpha_{2, \mathrm{~s}}\right\} 1+\mathrm{a}_{21, \mathrm{~s}} \mathrm{~L}_{1}^{3 / 2}\left(\mathrm{x}_{\mathrm{s}}^{2}\right)+\mathrm{a}_{22, \mathrm{~s}} \mathrm{~L}_{2}^{3 / 2}\left(\mathrm{x}_{\mathrm{s}}^{2}\right)\right]
\end{aligned}
$$

where $\alpha_{i, s}$ and $a_{i j, s}(i, j=1, k, i \leq j$, with $k \in N)$ are suitable constants to be uniquely determined (see Appendix D) from the previous condition of orthonormality.

By expanding the Spitzer function $\mathrm{G}_{\mathrm{s}}\left(\mathrm{H}_{\mathrm{s}}\right)$ in terms of the polynomials $\left\{\mathrm{K}_{\mathrm{j}, \mathrm{s}}\left(\mathrm{x}_{\mathrm{s}}^{2}\right), \mathrm{j} \in \mathrm{N}\right\}$ and retaining, as in References $[11,12]$, only the first three terms of the polynomial 
expansion, yields:

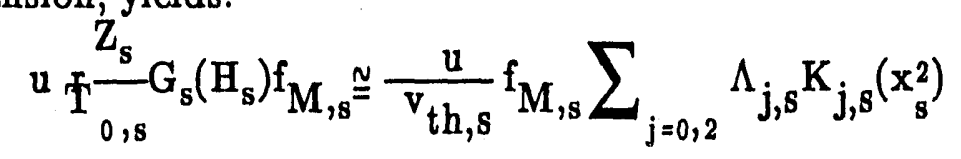

which gives, by substituting in Eq. (2.56), multiplying by $\frac{\mathrm{u}}{\mathrm{v}_{\mathrm{th}, \mathrm{s}}} \mathrm{K}_{\mathrm{j}, \mathrm{s}}\left(\mathrm{x}_{\mathrm{s}}^{2}\right)($ for $\mathrm{j}=0,2)$, and integrating in velocity space, gives the set of linear algebraic equations for the Fourier coefficients $\Lambda_{j, s}$ (for $j=0,2$ and $s=1, n$ ):

$$
\begin{aligned}
\Lambda_{j, s}= & -\frac{1}{2} \frac{Z_{s}}{T_{0, s}} \int^{3{ }^{3} v} \frac{u^{2}}{v_{t h, s}} f_{M, s} K_{j, s}\left(x_{s}^{2}\right)+ \\
& +\frac{1}{2} \sum_{\substack{k=1, n \\
k \neq s}} \int d^{3} v \frac{u}{v_{t h, s}} K_{j, s}\left(x_{s}^{2}\right) C_{s k}\left[f_{M, s} \mid \frac{2 u}{v_{t h, s}}\right. \\
& \left.f_{M, k} \sum_{j=0,2}^{\substack{k \neq s \\
j, k}} K_{j, k}\left(x_{k}^{2}\right)\right] .
\end{aligned}
$$

An approximate solution for the coefficients $\Lambda_{j, s}$ can be obtained by iteration; thus yielding, for example, for the first two iterations:

$$
\begin{aligned}
\Lambda_{j, s}^{(0)=} & -\frac{1}{2} \frac{Z_{s}}{T_{0, s}} \int d^{3} v \frac{u^{2}}{v_{t h, s}} f_{M, s} K_{j, s}\left(x_{s}^{2}\right) \\
\Lambda \int_{j, s}^{(1)}= & -\frac{1}{2} \frac{Z_{s}}{T_{0, s}} \int d^{3} v \frac{u^{2}}{v_{4} h, s} f_{M, s} K_{j, s}\left(x_{s}^{2}\right)+\frac{1}{2} \sum_{\substack{k=1, n \\
k \neq s}} \int d^{3} v \frac{u}{v_{t h, s}} \\
& K_{j, s}\left(x_{s}^{2}\right) C_{s k}\left[f_{M, s} \mid \frac{2 u}{v_{t h, s}} f_{M, k} \sum_{j=0,2} \Lambda_{j, k}^{(0)} K_{j, k}\left(x_{k}^{2}\right)\right] .
\end{aligned}
$$




\section{4 - CONSTRAINTS - TOTAL AND CLASSICAL FLUXES}

Solving the reduced gyrokinetic equation requires the determination of the electromagnetic field in terms of the Maxwell equations (taken in the quasi-static approximation), as well as suitabie constraints on the distributions function, resolving, in particular, the apparent undeterminacy related to the source terms depending on the relative toroidal angular frequency $\omega_{1,8}[3]$.

Let us investigate, in particular which constraints follow from the choice of the lowest-order distribution function $f_{M}$, s

Denoting $N_{s}=\int d^{3} v f_{s}(\underline{r}, \underline{v}, t)$ and $T_{\mathrm{s}}=\frac{1}{N_{s}} \int d^{3} v \frac{M_{s}}{2}(\underline{\mathbf{v}}-\underline{\mathbf{V}})^{2} f_{s}(\underline{r}, \underline{\underline{y}}, t)$ and, respectively, $\mathbb{N}_{s}=\mathbb{N}_{s}(\chi, \Psi, t)=, T_{0, s}=T_{0, s}(\Psi, t)$ the number density and temperature carried by the distribution functions $f_{s}(\underline{\underline{r}}, \underline{\underline{\mathbf{v}}}, \mathrm{t})$ and $\mathrm{f}_{\mathbf{M}, \mathbf{s}}(\underline{\mathbf{I}}, \underline{\mathbf{v}}, \mathrm{t})$, we impose the normalizations:

$$
\begin{aligned}
& <\mathrm{N}_{\mathrm{s}} / \eta_{\mathrm{s}}(\langle\omega\rangle)>=\overline{\mathrm{N}}_{\mathrm{s}} / \eta_{\mathrm{s}}(\langle\omega\rangle)=\mathrm{N}_{0, \mathrm{~s}}(\Psi, \mathrm{t}) \\
& <\mathrm{T}_{\mathrm{s}}>=\mathrm{T}_{0, \mathrm{~s}}(\Psi, \mathrm{t})
\end{aligned}
$$

where the factor $\eta_{\mathrm{s}}(\langle\omega\rangle)$ is defined by Eq. (2.23), whereas $\mathrm{N}_{0, \mathrm{~s}}$ is given in terms of Eq. (2.22). We notice that Eq. (4.1) is, of course, consistent with the requirements that $\mathrm{N}_{\mathrm{s}}=\overline{\mathrm{N}}_{\mathrm{s}}\left[1+\mathrm{O}\left(\varepsilon_{\mathrm{s}}\right)\right]$ and $\mathrm{T}_{\mathrm{s}}=\mathrm{T}_{0, \mathrm{~s}}\left[1+\mathrm{O}\left(\varepsilon_{\mathrm{s}}\right)\right]$.

Similarly, by expanding $\omega$ and $\Phi$, the electrostatic potential, in power seties of $\varepsilon$ (here $\varepsilon=\max \left\{\varepsilon_{\mathrm{s}}, \mathrm{s}=1, \mathrm{n}\right\}$ ), and denoting by $\omega_{0}$ and $\Phi_{-1}$ their leading-order contributions, one can always impose the normalizations:

$$
\begin{aligned}
& \langle\omega\rangle=\omega_{0}(\Psi, \mathrm{t}) \\
& \langle\Phi\rangle=\Phi_{-1}(\Psi, \mathrm{t}) .
\end{aligned}
$$

which, as a consequence of the requirement of existence of a rotating plasma equilibrium, implying $\omega_{0}=c \frac{\partial}{\partial \Psi^{\Phi}}-1$ [2], yield:

$$
\langle\omega\rangle=c \frac{\partial}{\partial \Psi}<\Phi>\text {. }
$$


An important problem which remains to be ascertained is whether the present equilibrium solution must fulfill further constraints, in particular constraints affecting the relative toroidal angular velocity $\omega_{1, \mathrm{~s}}$, besides those set by the transport ordering itself [see, e.g., Eqs. (2.7)-(2.11)].

To this purpose, we investigate the consistency with two basic requirements that must be fulfilled by the equilibrium distribution function $f_{M, s}$ as well as by the collisionless diamagnetic part of the distribution function, i.e., $g_{D_{s}}$, namely the condition of quasi neutrality and, moreover, the requirement of existence of a magnetostatic equilibrium, i.e., a (quasi-) static magnetic field $\underline{B}$ produced by the equilibrium diamagnetic current. Quasi neutrality is a consequence of the transport ordering and the assumption that $\left|\nabla^{2} \Phi / 4 \pi \Sigma_{s=1, n} Z_{s} e \bar{N}_{s}\right| \sim\left[\frac{\lambda}{L}\right]^{2} O(\varepsilon)$, with $\lambda_{D} / r_{L} \leq 1$ (where $\lambda_{D}$ is the Debye length and $r_{L}$ is the smallest Larmor radius among the particle species), and is expressed by the equation:

$$
\begin{aligned}
\Sigma_{s=1, n} Z_{s} e N_{s} & =\Sigma_{s=1, n} Z_{s} e N_{0, s} \exp \left\{\left[\frac{1}{2} M_{s} V^{2}-Z_{s} e[\Phi-\right.\right. \\
+ & \left.\left.\left.\frac{1}{c} \int_{0}^{\Psi} \mathrm{d} \Psi^{\prime}\langle\omega\rangle\right]\right] / T_{0, s}\right\}=0+O(\varepsilon)
\end{aligned}
$$

or, in terms of the reduced density $\mathrm{N}_{0, \mathrm{~s}}$ [see Eq. (2.22)], reads:

$$
\Sigma_{\mathrm{s}=1, \mathrm{n}} \mathrm{Z}_{\mathrm{s}} \mathrm{eN} \operatorname{los}_{0, \mathrm{~s}} \exp \left\{\left[\frac{1}{2} \mathrm{M}_{\mathrm{s}} \mathrm{V}^{2}-\mathrm{Z}_{\mathrm{s}} \mathrm{e}\left[\Phi-\frac{1}{\mathrm{c}} \int_{0}^{\Psi} \mathrm{d} \Psi^{\prime}<\omega_{\mathrm{s}}>\right]\right] / \mathrm{T}_{0, \mathrm{~s}}\right\}=0+\mathrm{O}(\varepsilon)
$$

which is an equation for the electrostatic potential $\Phi$. Letting $\Phi=\langle\Phi\rangle+\Phi$, thanks to Eq. (4.5), Eq. (4.7) gives an equation determining $\Phi$, i.e. :

$$
\mathrm{q}_{\mathrm{s}=1, \mathrm{n}} \mathrm{Z}_{\mathrm{s}} \mathrm{e} \xi_{\mathrm{s}} \exp \left\{-\mathrm{Z}_{\mathrm{s}} \mathrm{e} \tilde{\Phi} / \mathrm{T}_{0, \mathrm{~s}}\right\} \exp \left\{\left[\frac{1}{2} \mathrm{M}_{\mathrm{s}}<\omega>2\left[\mathrm{R}^{2-<\mathrm{R}^{2}>}\right]\right] / \mathrm{T}_{0, \mathrm{~s}}\right\}=0+\mathrm{O}(\varepsilon)
$$

with $\left.\xi_{\mathrm{s}} \equiv \mathrm{N}_{0, \mathrm{~s}} \exp \left\{\left[\frac{1}{2} \mathrm{M}_{\mathrm{s}}<\mathrm{R}^{2}>\langle\omega\rangle^{2}+\frac{\mathrm{Z} \mathrm{s}}{\mathrm{c}} \int_{0}^{\Psi} \mathrm{d}^{\prime} \omega_{1, \mathrm{~s}}\right]\right] / \mathrm{T}_{0, \mathrm{~s}}\right\} ;$ letting $\mathrm{e} \Psi / \mathrm{T}_{0, \mathrm{e}}=\ln \mathrm{Q}_{\mathrm{e}}$ and 
$\alpha_{s}=Z_{s} T_{0, e} / T_{0, s}$, one obtains in terms of $Q_{e}$ the algebraic equation:

$$
\mathrm{e} \xi_{\mathrm{e}} \mathrm{Q}_{\mathrm{e}}+\Sigma_{\mathrm{s}=1, \mathrm{n}_{\mathrm{s}} \mathrm{Z} \neq \mathrm{e}} \mathrm{Z}_{\mathrm{s}} \mathrm{Q}_{\mathrm{e}}^{\alpha}{ }_{\mathrm{s}}^{\mathrm{s}} \exp \left\{\left[\frac{1}{2} \mathrm{M}_{\mathrm{s}}<\omega>2\left[\mathrm{R}^{2}<\mathrm{R}^{2}>\right]\right] / \mathrm{T}_{0, \mathrm{~s}}\right\}=0+\mathrm{O}(\varepsilon)
$$

which generalizes the analogous equation yielding $\Phi$, in Reference [2], for a pure plasma. Hence, quasi-neutrality does not yield, per se, a constraint on $\omega_{1, s}$ but rather a relationship determining $\tilde{\Phi}$, and involving the reduced densities $\mathrm{N}_{0, \mathrm{~s}}$, with $\langle\omega\rangle$ and $T_{0, s}$ considered as prescribed functions of $\Psi$.

As shown in Appendix E, for a strongly rotating magnetoplasma the equation of magnetostatic equilibrium reads:

$$
(\nabla \times \underline{B}) \times \underline{B}=4 \pi[\nabla p+M N \underline{V} . \nabla \underline{\mathbf{V}}]
$$

where $p=\Sigma s=1, n N_{s} T_{0, s}$ is the scalar pressure and $M \bar{N}=\Sigma s=1, n M_{s} \bar{N}_{s}$.

On neglecting the displacement current (in view of the transport ordering), and using Ampere's law:

$$
\nabla \times \underline{B}=\frac{4 \pi}{\mathrm{C}} \underline{\mathrm{J}}
$$

where $\underline{\mathbf{J}}=\Sigma_{\mathrm{s}=1, \mathrm{n}} \mathrm{Z}_{\mathrm{s}} \mathrm{e} \int \mathrm{d}^{3} \mathrm{v} \underline{\mathbf{v f}}_{\mathrm{s}}(\underline{\mathbf{r}}, \underline{\mathbf{v}}, \mathrm{t})$ is the total current density vector, such an equation may be cast in the equivalent form:

$$
\underline{\mathbf{J}}_{\perp} \mathbf{x} \underline{\mathbf{B}}=\mathrm{c}[\nabla \mathrm{p}+\mathrm{M} \underline{\mathbf{N}} \cdot \nabla \underline{\mathbf{V}}] \text {. }
$$

Finally, we mention that Faraday's law, in view of the representation given by Eqs. (2.1) and (2.4) to the electromagnetic field, in terms of the vector and electrostatic potentials, is similarly fulfilled and does not yield additional constraints on the equilibrium [3].

It is also interesting to investigate under what conditions the g(neralized force $\mathrm{A}_{4, \mathrm{~s}}$ vanishes, i.e., when

$$
\frac{\partial}{\partial \Psi}\left[\frac{1}{T_{0, s}} \int_{0}^{\Psi} \mathrm{d} \Psi \omega_{1, \mathrm{~s}}\right]=0 \quad(\forall s=1, \mathrm{n})
$$

A particular case corresponds to requiring $\omega_{1, s}=0, \forall s=1, n$. In the case of equal tempe- 
ratures, this condition is equivalent to letting:

$$
\Delta \omega_{s \mathbf{k}} \equiv \omega_{1, \mathbf{s}}-\omega_{1, \mathbf{k}}=0 \quad(\forall \mathrm{s}, \mathrm{k}=1, \mathrm{n})
$$

which is, in turn, equivalent to maximizing the variational functional $W\left(k_{1} \mid k_{1}\right)$, under a suitable constraint. Let us inspect, in fact, the implications of the previous condition for a multispecies plasma; to this purpose, let us require

$$
\frac{\partial}{\partial \Delta \omega_{s k}} W\left(\mathbf{k}_{1} \mid \mathbf{k}_{1}\right)=0 \quad(\forall \mathrm{s}, \mathbf{k}=1, \mathbf{n})
$$

with the constraint

$$
\Delta \omega_{\mathrm{sk}}=\text { const. }(\forall \mathrm{s}, \mathrm{k}=1, \mathrm{n}) \text {. }
$$

Then there follows

$$
\Sigma_{s=1, n}\left\langle\int d^{3} M_{s} \underline{v} \cdot \hat{e}_{\vartheta} C_{s}\left(f_{M} \mid k_{1}+\bar{g}_{D}+G_{E}\right)\right\rangle=0
$$

which yields:

$$
\Delta \omega_{\mathrm{sk}}=0 \quad(\forall \mathrm{s}, \mathrm{k}=1, \mathrm{n}) .
$$

For a multispecies (and evell for a two-species) plasma this requirement may be, however, too stringent, since the forces $A_{4, s}$ in general need not exactly fulfill this constraint. Thus the possibility of nonvanishing contributions due to the generalized force $A_{4, s}$ cannot be ruled out a priori and the evaluation of the toroidal angular rotation velocity $\omega_{\mathrm{s}}$ must be performed.

This is a difficult task, at least in the framework of a variational theory, since the required moment of the distribution function, needed to determine $\omega_{\mathrm{s}}$, is not determined variationally at least using the present variational formulation. The velocity moment required, which has to be evaluated to first order in $O\left(\varepsilon_{\mathbf{s}}\right)$, is the toroidal mean velocity carried by the species $\mathrm{s}$, namely:

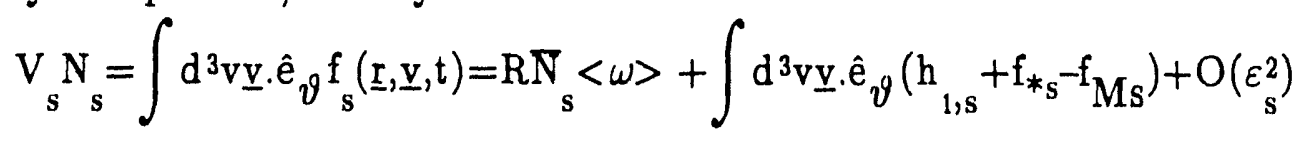

it follows 


$$
\omega_{s} \stackrel{N}{\stackrel{N}{N}} \mathbb{N}_{s}^{s}<\omega>+\frac{1}{R N} \int_{s} d^{3} v \underline{v} \cdot \hat{e}_{\vartheta}\left(h_{1, s}+f_{* s}-f_{M s}\right)
$$

and hence

$$
\omega_{1, s} \cong\langle\omega\rangle\left[\left\langle\frac{\mathbb{N}_{s}}{N_{s}}\right\rangle-1\right]+\left\langle\frac{1}{R N_{s}} \int d^{3} v \underline{v} \cdot \hat{e}_{\vartheta}\left(h_{1, s}+f_{*_{s}}-f_{M s}\right)\right\rangle
$$

where due to the normalization (4.1), $\left\langle\frac{N_{s}}{N_{s}}\right\rangle-1 \cong\left(\frac{N}{N_{s}, s}\right) \cong O\left(\varepsilon_{s}^{2}\right)$; such an equation can be used to evaluate the contributions to collisional transport due to the velocity perturbation associated with $\omega_{1, \mathrm{~s}}$.

To obtain order-of-magnitude estimates for $\omega_{1, \mathrm{~s}}$ in the case of a multi-species plasma, an approximate solution based on the approximate collision operator due to Hirshman and Sigmar can be used. For example, considering, for definitess, the case of a weakly collisional plasma with vanishing baricentric toroidal rotation and toroidal differential rotation $\left(\langle\omega\rangle=0\right.$ and $A_{3, s}=0$ ), the approximate analytic solution obtained for $\mathrm{f}_{1, k}$ by Hirshman, Sigmar and Clarke [13] can be used:

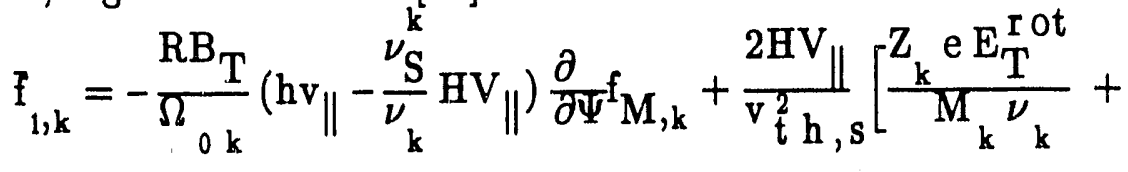

$$
\begin{aligned}
& \left.+\sum_{j=1, n} \frac{\nu_{\mathrm{S}}^{k j}}{\nu_{k}} \overline{\mathrm{r}}_{j k}\right] \mathrm{f}_{\mathrm{M}_{, k}}
\end{aligned}
$$

where $\nu_{\mathrm{S}}^{\mathrm{k}}$, and $\nu_{k}$ are suitable effective collision frequencies and $\overline{\mathrm{r}}_{\mathrm{jk}}$ momentum restoring coefficients; moreover, $\Omega_{0 k}=Z_{k} e<B_{2}>V^{2} / M_{k} c, H=\Theta\left(1 / B_{\max }-\lambda\right), h=<B_{2}>V^{2} / B$ and

$$
\mathrm{V}_{\|}=\frac{\mathrm{v} \sigma}{2}\left\langle\mathrm{~B}^{2}\right\rangle^{1 / 2} \int_{\lambda}^{1 / \mathrm{B}_{\max }} \mathrm{d} \lambda(1-\lambda \mathrm{B})^{-1 / 2}
$$

with $\sigma=\operatorname{sign}\left(\mathbf{v}_{\|}\right), \mathbf{v} \|=\mathbf{v} \cdot \underline{\mathbf{b}}$, while the velocity-space variables used are $\mathrm{v}, \lambda=2 \mu / \mathrm{v}^{2}$ and a suitable gyrophase. 
Then the relative toroidal angular velocity $\omega_{1, \mathbf{k}}$ can be computed substituting Eq. (4.22) in Eq. (4.21). Then, neglecting for definitness the inductive eloctric field and assuming large aspect ratios (i.e., $\left.\delta \equiv O\left(\left(r / R_{0}\right)^{1 / 2}\right)<<1\right)$, one finds that:

$$
\omega_{1, \mathbf{k}} \sim O(\delta)
$$

which means that the constributions to the neoclassical fluxes carried by the perturbations $\omega_{1, \mathbf{k}}$ are of higher order in the inverse aspect-ratio in comparison to the leading-order contributions. This conclusion implies that the evaluation of such corrections, at least in the example considered, should be relevant only for estimating finite aspect-ratio effects.

Finally, let us turn to the problem of determining the (total) fluxes for the relevant dynamical variables.

To this end, let us recall that in order to obtain a complete macroscopic description cf a strongly rotating magnetoplasma, the moment equations needed are those yieldir $g$ particle number conservation (equation of continuity), energy balance and angular momentum balance $[1,2]$.

Hence the relevant fluxes to be determined are the particle,"energy" and angular momentum fluxes:

$$
\Gamma_{\mathrm{X}, \mathrm{s}}=\left\langle\nabla \Psi \cdot \int \mathrm{d}^{3} \mathrm{v} \mathrm{X}_{\mathrm{s}}^{\mathrm{v}} \cdot \mathrm{f}, \mathrm{s}\right\rangle
$$

where, respectively, $\mathrm{X}_{\mathrm{s}}=1, \mathrm{H}_{\mathrm{s}}, \mathrm{P}_{\mathrm{T}, \mathrm{s}}=\mathrm{M}_{\mathrm{s}} \mathrm{R} \boldsymbol{\mathrm { v }} \cdot \hat{\mathrm{e}}_{\vartheta}$

As shown in a previous work [2], suitable moment equations can be used to obtain integral representations for all of the previous fluxes, including the previously defined "neoclassical" contributions given earlier in Sec. 2. However, in this case, the method will be used to determine the explicit contributions to the fluxes, i.e., the so-called classical fluxes.

To this end, we first construct suitable moments of the Fokker-Planck kinetic equation. Hence if $X_{s}=X_{s}(\underline{r}, \mathbb{Y}, t)$ is assumed sufficiently smooth and integrable one obtains for $\mathrm{X}_{\mathrm{s}}$ the moment equation: 


$$
\begin{gathered}
\left\langle\frac{\partial}{\partial t} \int d^{3} \mathrm{vX}_{s} f_{s}\right\rangle+\left(V^{\prime}\right)^{-1} \frac{\partial}{\partial \Psi}\left[V^{\prime}\left\langle\nabla \Psi \cdot \int_{d^{3} v X_{s} \cdot f} \cdot f_{s}\right\rangle=\right. \\
=\left\langle\int d^{3} v\left[f_{s} \frac{d}{d t} X_{s}+X_{s} C_{s}(f \mid f)\right]\right\rangle .
\end{gathered}
$$

Since by assumption, i.e., thanks to the transport ordering, the left hand side of the previous equation vanishes up to second order in $\varepsilon$, it follows that, to the same order there results:

$$
\left\langle\int d^{3} v\left[f_{s} \frac{d}{d t} x_{s}+X_{s} C_{s}(f \mid f)\right]\right\rangle=0 .
$$

Hence selecting $\mathrm{X}_{\mathrm{s}}$ in a suitable way, more precisely as $\mathrm{X}_{\mathrm{s}}=\mathrm{H}_{\mathrm{s}}, \mathrm{P}_{\mathrm{T}, \mathrm{s}}, \mathrm{P}_{\mathrm{T}, \mathrm{s}}^{2}$, and $\mathrm{X}_{\mathrm{s}}=\mathrm{H}_{\mathrm{s}} \mathrm{P}_{\mathrm{T}, \mathrm{s}}$, one obtains following the method of Reference [2], the representation:

$$
\Gamma_{\mathrm{X}, \mathrm{s}}=\Gamma_{\mathrm{X}, \mathrm{s}}^{(\mathrm{c})}+\Gamma_{\mathrm{X}, \mathrm{s}}^{(\mathrm{n})} \quad\left(\mathrm{X}_{\mathrm{s}}=1, \mathrm{H}_{\mathrm{s}}, \mathrm{P}_{\mathrm{T}, \mathrm{s}}=\mathrm{M}_{\mathrm{s}} \mathrm{R}_{\mathbf{Z}} \cdot \hat{e}_{\vartheta}\right)
$$

where $\Gamma_{X, s}(n)$ are the neoclassical fluxes defined previously [see Eqs.(2.96) and (2.97)], whereas $\Gamma_{\mathrm{X}}^{(\mathrm{c})}$, to be denoted as "classical" fluxes, read simply:

$$
\Gamma_{X, s}^{(c)}=\frac{M_{s} c}{Z_{s}^{e}}\left\langle R \int d^{3} v X_{s} \underline{w} \cdot \hat{e}_{\vartheta} C_{s}\left(f_{M} \mid \tilde{g}_{D}\right)\right\rangle \text {. }
$$




\section{5 - CONCLUDING REMARKS}

A general formalism has been formulated for the investigation of a strongly rotating toroidal magnetoplasma in a transport regime. The gyrokinetic equation obtained, which advances in time the kinetic distribution function, has been given a reduced form particularly suited for transport applications, in particular for the investigations of multiple-species transpor $t$ in the presence of inductive effects.

The main results of the paper can be summarized as follows:

1) the gyrokinetic kinetic equation has been expressed in terms of explicit velocity perturbations driven by toroidal rotation effects, in particular toroidal relative rotation speed with respect to the center of mass velocity, which account for an additional shear effect previously ignored in collisional transport theory;

2) all source terms appearing in the gyrokinetic equation have been brought inside the collision operator, thus yielding a reduced form of the equation which is particularly useful for transport theory calculations; previously such a form of the equation was obtained only in special cases, as in Reference [9] by Rosenbluth et al., where this transformation was actually carried out only for the so-called banana regime; or in Reference [2] by Catto et al., where, however, the Spitzer source term was not elaborated further;

3) a constrained variational formulation has been given for the gyrokinetic equation, which is valid for arbitrary plasma collisionalities and exhibits simple relationships with the entropy production rate and the neoclassical fluxes; as an application integral expressions have been given for the neoclassical fluxes;

4) the constraints to be fulfilled by the equilibrium distribution function have been investigated and, in particular, the compatibility with the existence of velocity perturbations driven by toroidal angular velocity, relative to the center of mass of the fluid element, has been ascertained;

5) finally, an auxiliary multiple-species Spitzer problem has been formulated in terms of the exact Fokker-Planck collision operator. The resulting integro-differential equation for the Spitzer function has been solved analytically in terms of an expansion in orthonormal polynomials, yielding an iterative approximation to the Spitzer function. 


\section{ACKNOWLEDGMENTS}

This work has been sponsored in part by the Italian National Research Council, through the "Gruppo Nazionale per la Fisica Matematica del CNR", the Princeton Plasma Physics Laboratory, U.S. Department of Energy Contract \#DE-AC02-76CHO -3073 and the "Consorzio di Magnetofluidodinamica", Trieste University, Italy.

M.Tessarotto was privileged for the hospitality of the Princeton Plasma Physics Laboratory during this research.

The authors are indebted to Prof. Ira B. Bernstein (Yale University, New Haven, Conn., USA) for useful comments. 


\section{REFERENCES}

1 - F.L. Hinton and S.K. Wong, Phys.Fluids 28, 3082 (1985).

2 - P.J. Catto, I.B. Bernstein and M. Tessarotto, Phys.Fluids $\underline{30}, 2784$ (1987).

3 - P.J. Catto and M. Tessarotto, Rarefied Gas Dynamics, ed.by E.P.Muntz, D.P. Weaver and D.H. Campbell, Vol.116, p.89, AIAA Washington DC (1989).

4 - M.N. Rosenbluth, P.H. Rutherford, J.B. Taylor, E.A. Frieman and

L.M. Kovrizhnik, Plasma Phys. and Controlled Nuclear Fusion Research 1970, Proc. of the Fourth International Conference (IAEA,Vienna,1971), Vol.I,p.495.

5 - R.D. Hazeltine, Phys.Fluids, $\underline{17}, 961$ (1974).

6 - K.T. Tsang and E.A. Frieman, Phys.Fluids, $\underline{19}, 747$ (1976).

7 - S.K. Wong and K.H. Burrel, Phys.Fluids $\underline{25}, 1863$ (1982).

8 - S.K. Wong and F.L. Hinton, Phys.Rev.Lett. 52, 827 (1984).

9 - M.N. Rosenbluth, R.D. Hazeltine and F.L. Hinton, Phys.Fluids $\underline{15}$, 116 (1972).

10 - M. Tessarotto, N.Cimento $\underline{75 B}, 19$ (1983).

11 - M. Tessarotto and R. White, Princeton Plasma Physics Laboratory Internal Report, to appear (1991).

12 - S.P. Hirshman, Phys.Fluids $\underline{20}, 589$ (1977).

13 - S.P. Hirshman, Phys.Fluids 21, 1295 (1978).

14 - S.P. Hirshman and D.J. Sigmar, Phys.Fluids $\underline{19}, 1532$ (1976)

15 - S.P. Hirshman,D.J. Sigmar and J.F. Clarke, Phys.Fluids $\underline{19}, 656$ (1976). 
APPENDIX A: LINEARIZED FOKKER-PLANCK COLLISION OPERATOR FOR THE MULTISPECIES SPITZER PROBLEM

Letting $\mathrm{K}_{\mathbf{s}}=\mathrm{K}_{\mathrm{s}}\left(\mathrm{x}_{\mathrm{s}}\right)$ be a smooth and integrable function, with $\mathrm{x}_{\mathrm{s}}=\mathrm{v}^{\prime} / \mathrm{v}_{\text {th, }}$ and $\mathrm{C}_{\mathbf{s}}\left(\mathrm{f}_{\mathrm{M}} \mid \mathrm{uKf}_{\mathrm{M}}\right)$ the Fokker-Planck collision operator, let us denote:

$$
\mathrm{C}_{\mathbf{s}}\left(\mathrm{f}_{\mathrm{M}} \mid \mathrm{uKf}_{\mathrm{M}}\right)=\mathrm{uI} I_{1, \mathrm{~s}}\left\{\mathrm{Kf}_{\mathrm{M}_{, \mathrm{s}}}\right\}+\mathrm{u} I_{2, \mathrm{~s}}\left\{\mathrm{Kf}_{\mathrm{M}}\right\}
$$

with $I_{i, s}(\mathrm{i}=1,2)$ the linear operators:

$$
\begin{aligned}
I_{1, \mathrm{~s}}\left\{\mathrm{Kf}_{\mathrm{M}, \mathrm{s}}\right\} & =\sum_{\mathrm{k}=1, \mathrm{n}} \mathrm{C}_{\mathrm{sk}}\left(\mathrm{f}_{\mathrm{M}_{, \mathrm{k}}} \mid \mathrm{uK} \mathrm{f}_{\mathrm{s}} \mathrm{M}, \mathrm{s}_{\mathrm{s}}\right) / \mathrm{u} \\
I_{1, \mathrm{~s}}\left\{\mathrm{Kf}_{\mathrm{M}_{, \mathrm{s}}}\right\} & =\sum_{\mathrm{k}=1, \mathrm{n}} \mathrm{C}_{\mathrm{sk}}\left(\mathrm{f}_{\mathrm{M}, \mathrm{s}} \mid \mathrm{uK} \mathrm{f}_{\mathrm{s}} \mathrm{M}_{, \mathrm{k}}\right) / \mathrm{u} .
\end{aligned}
$$

There results, in particular [11]:

$$
I_{1, \mathrm{~s}}\left\{\mathrm{Kf}_{\mathrm{M}, \mathrm{s}}\right\}=-\frac{1}{2} \frac{\mathrm{U}_{\mathrm{s}}^{(1)}}{\mathrm{v}^{\prime 2}} \mathrm{~K}_{\mathrm{s}}+\frac{1}{\mathrm{v}^{\prime 3}} \frac{\partial}{\partial \mathrm{v}^{\prime}},\left\{\mathrm{v}^{\prime 2}\left(\mathrm{U}_{\mathrm{s}}^{(1)}-\mathrm{U}_{\mathrm{s}}^{(2)}\right) \frac{\partial}{\partial \mathrm{v}^{\prime}},\left(\mathrm{v}^{\prime} \mathrm{K}_{\mathrm{s}}\right]\right\}
$$

where $\mathrm{U}_{\mathrm{s}}^{(1)}, \mathrm{U}_{\mathrm{s}}^{(2)}$ are the energy integrals:

$$
\begin{aligned}
& \mathrm{U}_{\mathrm{s}}^{(1)}=\mathrm{f}_{\mathrm{M}, \mathrm{s}} \pi \sum_{\mathrm{k}=1, \mathrm{n}} \mathrm{q}_{\mathrm{sk}} \mathrm{v}_{\mathrm{th}, \mathrm{k}}^{2}\left\{\left[2-3 \mathrm{x}_{\mathrm{k}}^{-2}\right] \int_{0}^{\mathrm{x}} \mathrm{dyf}_{\mathrm{M}, \mathrm{k}}^{\mathrm{k}}(\mathrm{y})+3 \mathrm{x}_{\mathrm{k}}^{-2 f} \mathrm{M}, \mathrm{k}_{\mathrm{k}}\left(\mathrm{x}_{\mathrm{k}}\right)\right\} \\
& \underset{\mathrm{s}}{\mathrm{U}^{(2)}}=\mathrm{f}_{\mathrm{M}, \mathrm{s}} \pi \sum_{\mathrm{k}=1, \mathrm{n}} \mathrm{q}_{\mathrm{sk}} \mathrm{v}_{\mathrm{th}, \mathrm{k}}^{2}\left\{\left[2-\mathrm{x}_{\mathrm{k}}^{-2}\right] \int_{0}^{\mathrm{dyf}_{\mathrm{M}, \mathrm{k}}^{\mathrm{k}}}(\mathrm{y})+\mathrm{x}_{\mathrm{k}}^{-2} \mathrm{f}_{\mathrm{M}, \mathrm{k}}\left(\mathrm{x}_{\mathrm{k}}\right)\right\}
\end{aligned}
$$

and $\mathrm{q}_{\mathrm{sk}}=2 \pi \mathrm{Z}_{\mathrm{s}}^{2} \mathrm{Z}_{\mathrm{k}}^{2} \mathrm{e}^{4} \ln \Lambda / \mathrm{M}_{\mathrm{s}}^{2}$ 


\section{APPENDIX B: ENTROPY PRODUCTION RATE}

Starting from the the definition of kinetic entropy

$$
S=-\int d^{3} v \sum_{s=1, n} f \ln f_{s}
$$

the entropy production rate reads:

$$
\frac{d}{d t} S=-\int d^{3} v \sum_{s=1, n} C_{s}(f \mid f) \ln f .
$$

Using the transport ordering and expanding in the Larmor radius $\ln _{\mathrm{s}}$

$$
\operatorname{lnf} f_{s}=\operatorname{lnf} f_{M, s}+\varepsilon f_{s 1, s} / f_{M, s}+O\left(\varepsilon_{s}^{2}\right)
$$

as well as the collision operator, there results, neglecting contributions of higher order:

$$
\frac{d}{d t} S=-\int d^{3} v \Sigma_{s=1, n} C\left(f_{M} \mid h_{1}+f_{*}-f_{M}\right)\left[\ln f_{M, s}+f_{1, s} / f_{M, s}\right]
$$

where

$$
\operatorname{lnf} \mathrm{M}_{\mathrm{M}, \mathrm{s}}=\ln N_{0, \mathrm{~s}}-\frac{3}{2} \ln \mathrm{T}_{0, \mathrm{~s}}-\mathrm{H}_{\mathrm{s}} / \mathrm{T}_{0, \mathrm{~s}}+\text { const., }
$$

and according to Eq. (2.86) we have denoted:

$$
N_{0, \mathrm{~s}}=\mathrm{N}_{0, \mathrm{~s}}(\Psi, \mathrm{t}) \exp \left\{\frac{\mathrm{Z}_{\mathrm{s}} \mathrm{e}}{\mathrm{cT} \mathrm{T}_{0, \mathrm{~s}}} \int_{0}^{\Psi} \mathrm{d} \Psi^{\prime} \omega_{1, \mathrm{~s}}\right\}
$$

Requiring

$$
\int d^{3} v \Sigma_{s=1, n} H_{s} C_{s}\left(f_{M} \mid h_{1}+f_{*}-f_{M}\right) / T_{0, s} \sim O\left(\varepsilon_{s}^{3}\right)
$$

it follows:

$$
\left\langle\frac{d}{d t} s\right\rangle=\left\langle\int d^{3} v \Sigma_{s=1, n} C_{s}\left(f_{M} \mid h_{1}+f_{*}-f_{M}\right) f_{1, s} / f_{M, s}\right\rangle .
$$

Letting $\mathrm{f}_{\mathbf{s}}=\mathrm{f}_{\mathbf{s}}+\mathrm{f}_{\mathrm{s}}$, one obtains the relationship between the surface-averaged entropy production rate $\left\langle\frac{\mathrm{d}}{\mathrm{dt}} \mathrm{s}\right\rangle$ and the variational functional $\mathrm{W}\left(\mathbf{k}_{1} \mid \mathbf{k}_{1}\right)$ [see Eq. (2.87)]:

$$
\left\langle\frac{\mathrm{d}}{\mathrm{dt}} \mathrm{s}\right\rangle=-\mathrm{W}\left(\mathrm{k}_{1} \mid \mathrm{k}_{1}\right)+\left\langle\left.\frac{\mathrm{d}}{\mathrm{dt}} \mathrm{s}\right|_{\text {classical }}\right\rangle
$$

where $\left\langle\left.\frac{\mathrm{d}}{\mathrm{dt}} \mathrm{S}\right|_{\text {classical }}\right\rangle \equiv\left\langle\int \mathrm{d}^{3} \mathrm{v} \sum_{\mathrm{s}=1, \mathrm{n}} \mathrm{C}_{\mathrm{s}}\left(\mathrm{f}_{\mathrm{M}} \mid \tilde{\mathrm{g}}_{\mathrm{D}}\right) \tilde{\mathrm{g}}_{\mathrm{D}_{\mathrm{s}}} / \mathrm{f}_{\mathrm{M}, \mathrm{s}}\right\rangle$ and can be expressed as a linear function of the classical fluxes [see. Eq. (4.29)]. 


\section{APPENDIX C: DIFFERENT TEMPERATURES VARIATIONAL PRINCIPLE}

Let us consider a multispecies plasma assuming that some species $\mathrm{s}$ (electrons and heavy impurities) may have disparate masses with respect to each other or some ionic species $i$, in the sense that either $M_{s} / M_{i}>>1$ or $<<1$ with $\varepsilon=s_{1}, \ldots, s_{n}$. Then it is easily proven, by introducing a suitable subsidiary expansion in the small mass ratios, that approximate rotating Maxwellian equilibria of the type defined by Eq. (2.16) exist which are each characterized locally by a different temperature $\mathrm{T}_{0, \mathrm{~s}}=\mathrm{T}_{0, \mathrm{~s}}(\Psi, t)$ $\left(\mathrm{s}=\mathrm{s}_{1}, \ldots, \mathrm{s}_{\mathrm{n}}\right)$, with $\mathrm{T}_{0, \mathrm{~s}}(\Psi, \mathrm{t}) \neq \mathrm{T}_{0}(\Psi, \mathrm{t})$ and for all species $\mathrm{k}$ (i.e., $\left.\mathrm{k}=1, \mathrm{n}\right)$ fulfilling the orderings

$$
\begin{aligned}
& \left.\left\{\mathrm{T}_{0, \mathbf{s}}-\mathrm{T}_{0, \mathbf{k}}\right)\right\} / \mathrm{T}_{0} \sim \mathrm{O}\left(\varepsilon^{0}\right) \\
& \mathbf{M}_{\mathbf{s}} / \mathrm{M}_{\mathrm{i}} \sim \mathrm{O}\left(\varepsilon_{\mathbf{s}}\right) \text { or } \mathrm{M}_{\mathrm{i}} / \mathrm{M}_{\mathrm{s}} \sim \mathrm{O}\left(\varepsilon_{\mathbf{s}}\right)
\end{aligned}
$$

with $\mathrm{T}_{0}$ denoting the temperature carried, at least, by one remaining particle species (i).

To extend the constrained variational formulation given by Euler equation [Eq. (2.91)] together with the constraints Eq. (2.92), it is sufficient to change in a suitable way the definition of the scalar product Eq. (2.90), and consequently of all the related functionals, i.e., $W\left(k_{1} \mid k_{1}\right)$ and $Q_{i}\left(k_{1} \mid k_{1}\right)(i=1,2)$, defined by Eqs. (2.87)-(2.89). To this purpose, let us introduce, in place of Eq. (2.90), the definition for suitable vector functions $A=\left\{A_{j}(\underline{r}, \underline{v}, t), j=1, n\right\}, B=\{B(\underline{r}, \underline{v}, t), j=1, n\}$

$$
\langle A \mid B\rangle=\Sigma_{s=1, n} \frac{T_{0, s}}{T_{0}}\left\langle\int d^{3} v_{s} A_{s} / f_{M}, s\right.
$$

and redefine in terms of it the aforementioned functionals. It is then easily proven that, correct to leading order in the small mass ratios (i.e., either $\mathrm{M}_{\mathrm{s}} / \mathrm{M}_{\mathrm{i}}<<1$ or $\mathrm{M}_{\mathrm{i}} / \mathrm{M}_{\mathrm{s}}<<1$ ) the linearized Fokker-Planck collision operator is symmetric with respect to the scalar product (C.1), namely :

$$
<\mathrm{A}\left|\mathrm{C}\left(\mathrm{f}_{\mathrm{M}} \mid \mathrm{B}\right)>=<\mathrm{B}\right| \mathrm{C}\left(\mathrm{f}_{\mathrm{M}} \mid \mathrm{A}\right)>\text {. }
$$

It follows that, in terms of the present definition of the scalar product and correct to 
order $\mathrm{O}\left(\left(\mathrm{M}_{\mathrm{s}} / \mathrm{M}_{\mathrm{i}}\right)^{\mathscr{V} /}\right.$ if $\mathrm{M}_{\mathrm{s}} / \mathrm{M}_{\mathrm{i}}<<1$ (or, respectively, to order $\mathrm{O}\left(\left(\mathrm{M}_{\mathrm{i}} / \mathrm{M}_{\mathrm{s}}\right)^{\mathrm{V} 2}\right.$ ) if $\mathrm{M}_{\mathrm{i}} / \mathrm{M}_{\mathrm{s}}<<1$ ) Eqs. (2.91) and (2.92) yield a constrained variational formulation for the reduced kinetic equation [Eq. (2.77)], in the case of rotating Maxwellian equilibria having different temperatures and in the ordering specified by Eqs. (C.1), (C.2).

As a consequence, it follows that the extremal value of the functional $W\left(k_{1} \mid k_{1}\right)$, defined by Eq. (2.94), reads now:

$$
\begin{aligned}
& \mathrm{W}\left(\mathrm{k}_{1} \mid \mathrm{k}_{1}\right)=\Sigma_{\mathrm{s}=1, \mathrm{n}} \frac{\mathrm{T}}{\mathrm{T}_{0}, \mathrm{~s}}\left[\left(\mathrm{~A}_{1, \mathrm{~s}}+\mathrm{A}_{4, \mathrm{~s}}\right) \Gamma_{1, \mathrm{~s}}^{(\mathrm{n})}+\mathrm{A}_{2, \mathrm{~s}} \Gamma_{\mathrm{H}, \mathrm{s}}^{(\mathrm{n})}+\right. \\
& \left.\quad+\mathrm{A}_{3, \mathrm{~s}} \mathrm{~L}_{\mathrm{s}}^{(\mathrm{n})}-\left\langle\mathrm{J}_{\| \mathrm{s}} \underline{\mathrm{b}} \cdot(\underline{\mathrm{E}}+\nabla \Phi) / \mathrm{T}_{0, \mathrm{~s}}\right\rangle\right] .
\end{aligned}
$$




\section{APPENDIX D: ORTHONORMAL POLYNOMIALS $\left\{\mathrm{K}_{\mathrm{j}, \mathrm{s}}\left(\mathrm{x}_{\mathrm{s}}{ }_{\mathrm{s}}\right), \mathrm{jEN}\right\}$}

In order to define the orthonormal basis $\left\{\mathrm{K}_{\mathrm{j}, \mathrm{s}}\left(\mathrm{x}_{\mathrm{s}}^{2}\right), j \in \mathrm{N}\right\}$, we need to evaluate the coefficients $\alpha_{i, s}$ and $a_{i j, s}(i, j=1, k, i \leq j$, with $k \in N)$ appearing in their definition, by making use of the orthonormality conditions Eq. (3.6).

For examples, let us evaluate the previous coefficients for the first three polynomials. For this purpose, let us define the matrix elements $\mathrm{M}_{\mathrm{sk}}^{\mathrm{ij}}$ and $\mathrm{N}_{\text {sk }}^{\mathrm{ij}}$ (with $\mathrm{s}, \mathrm{k}=1, \mathrm{n}$ and $\mathrm{i}, \mathrm{j} \in \mathrm{N})^{11}$ :

$$
\begin{aligned}
& M_{\text {sk }}^{i j}=\int d^{3} v \frac{u}{v_{t h, s}} J_{j}^{3 / 2}\left(x_{s}^{2}\right) C_{s k}\left(f_{M, s} \mid 2 \frac{u}{v_{t h}, k} L_{j}^{3 / 2}\left(x_{k}^{2}\right) f_{M, k}\right) \\
& N_{s k}^{i j}=\int d^{3} v \frac{u}{v_{t h, s}} L_{j}^{3 / 2}\left(x_{s}^{2}\right) C_{s k}\left(2 \frac{u}{v_{t h, s}} L_{j}^{3 / 2}\left(x_{s}^{2}\right) f_{M, s} \mid f_{M, k}\right) .
\end{aligned}
$$

There results from the definitions Eqs. $(65)-(67)$ and using the condition orthonormality Eq. (64):

$$
\alpha_{0, s}=\left[\left|M_{s}^{00}\right|\right]^{i / 2}
$$

with $\mathrm{M}_{\mathrm{s}}^{00}=\sum_{\mathrm{k}=1, \mathrm{n}} \mathrm{M}_{\mathrm{sk}}^{00}$, and hence $\mathrm{K}_{0,8}\left(\mathrm{x}_{\mathrm{s}}^{2}\right)=\left(\left|\mathrm{M}_{\mathrm{s}}^{00}\right|\right)^{1 / 2}$. Similarly to determine $\mathrm{K}_{1, \mathrm{~s}}\left(\mathrm{x}_{\mathrm{s}}^{2}\right)$, i.e., the coefficients $\alpha_{1, \mathrm{~s}}$ and $a_{11, \mathrm{~s}}$ it is sufficient to use the condition of orthogonality between $\mathrm{K}_{0, \mathrm{~s}}\left(\mathrm{x}_{\mathrm{s}}^{2}\right)$ and $\mathrm{K}_{1, \mathrm{~s}}\left(\mathrm{x}_{\mathrm{s}}^{2}\right)$ as well as the normalization for $\mathrm{K}_{1, \mathrm{~s}}\left(\mathrm{x}_{\mathrm{s}}^{2}\right)$, i.e.,

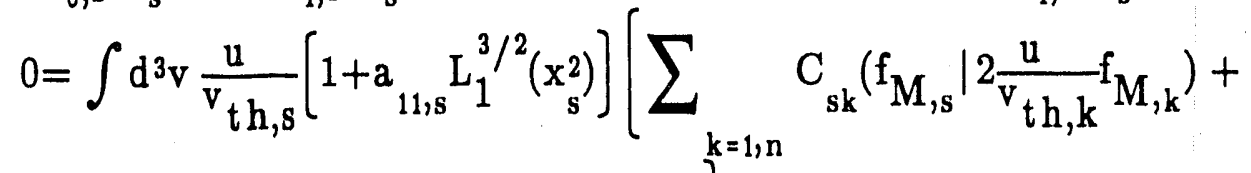

$$
\begin{aligned}
& \left.\mathrm{C}_{\mathrm{ss}}\left(\mathrm{f}_{\mathrm{M}, \mathrm{s}} \mid 2 \frac{\mathrm{u}}{\mathrm{v}_{\mathrm{th}, \mathrm{s}}} \mathrm{f}_{\mathrm{M}, \mathrm{s}}\right)\right]^{\mathrm{k}=1, \mathrm{n}}
\end{aligned}
$$

and, respectively:

which deliver:

$$
\begin{gathered}
1=-\int \mathrm{d}^{3} \mathrm{v} \frac{\mathrm{u}}{\mathrm{v}_{\mathrm{th}, \mathrm{s}}} \mathrm{K}_{1, \mathrm{~s}}\left(\mathrm{x}_{\mathrm{s}}^{2}\right)\left[\sum_{\mathrm{k}=\mathrm{l}, \mathrm{n}} \mathrm{C}_{\mathrm{sk}}\left(\mathrm{f}_{\mathrm{M}, \mathrm{s}} \mid 2 \frac{\mathrm{u}}{\mathrm{v}_{\mathrm{th}, \mathrm{k}}} \mathrm{K}_{1, \mathrm{k}}\left(\mathrm{x}_{\mathrm{k}}^{2}\right) \mathrm{f}_{\mathrm{M}, \mathrm{k}}\right)+\right. \\
\left.\mathrm{C}_{\mathrm{ss}}\left(\mathrm{f}_{\mathrm{M}, \mathrm{s}} \mid 2 \frac{\mathrm{u}}{\mathrm{v}_{\mathrm{th}, \mathrm{s}}} \mathrm{K}_{1, \mathrm{~s}}\left(\mathrm{x}_{\mathrm{s}}^{2}\right) \mathrm{f}_{\mathrm{M}, \mathrm{s}}\right)\right]
\end{gathered}
$$




$$
\begin{aligned}
& \alpha_{1,8}=\left[\left|\left[\frac{M_{s}^{00}}{M_{s}^{10}}\right]^{2} M_{s}^{11}-M_{s}^{10}\right|\right]^{1 / 2} \\
& a_{11,8}=-\frac{M_{s}^{00}}{M_{s}^{10}} .
\end{aligned}
$$

Analogously the coefficients of $\mathrm{K}_{2,}, \mathrm{~s}_{\mathbf{s}}\left(\mathrm{x}^{2}\right)$ are:

$$
\begin{aligned}
a_{21, s} & =-\frac{M_{s}^{00}}{M_{s}^{10}}-a_{22, s} \frac{M_{s}^{20}}{M_{s}^{10}} \\
a_{22, s} & =\frac{M_{s}^{01}-M_{s}^{11} M_{s}^{00} / M_{s}^{10}}{M_{s}^{21}-M_{s}^{11} M_{s}^{20} / M_{s}^{10}} \\
\alpha_{2, s} & =\left[\left|a_{22, s}\left[M_{s}^{20}+M_{s}^{21, a}+M_{21, s}^{22 a} a_{22, s}\right]\right|\right]^{1 / 2} .
\end{aligned}
$$




\section{APPENDIX E: GENERALIZED EQUATION OF MAGNETOSTATIC EQUILIBRIUM AND GENERALIZED GRAD-SHAFRANOV EQUATION}

In order to derive Eq. (4.10), i.e., the generalized equation of magnetostatic equilibrium, :nd to prove the identity Eq. (4.12), let "s evaluate the linear momentum moment of the Fokker-Planck kinetic equation, yielding after summation over species the momentum balance equation:

$$
\Sigma_{s=1, n} M_{s} \int d^{3} v \underline{v} \frac{d}{d t}\left(f_{*_{s}}+h_{s}\right)=0
$$

Forming the identity

$$
\begin{aligned}
& \Sigma_{s=1, n} M_{s} \int d^{3} v \underline{v} \frac{d}{d t}\left(f_{*_{s}}+h_{s}\right)=\frac{\partial}{\partial t} \Sigma_{s=1, n} M_{s} \int d^{3} v \underline{v}\left(f_{*_{s}}+h_{s}\right)+ \\
& \nabla \cdot \Sigma_{s=1, n} M_{s} \int d^{3} v \underline{v v}\left(f_{*_{s}}+h_{s}\right)-\Sigma_{s=1, n} Z_{s} e \int d^{3} v\left(\underline{E}+\frac{\underline{v}}{c} x \underline{B}\right)\left(f_{*_{s}}+h_{s}\right)
\end{aligned}
$$

in view of the transport ordering [Eqs. (2.7)-(2.11)], it gives to leading order:

$$
\Sigma_{s=1, n} M_{s} \int d^{3} v \underline{v} \frac{d}{d t}\left(f_{* s}+h\right)=\nabla \cdot \underline{\underline{S}}-\underline{E} \Sigma_{s=1, n} Z_{s} e N_{s}-\frac{1}{c} \underline{J} x \underline{B}=0
$$

namely, invoking quasi neutrality:

$$
\nabla \cdot \underline{\underline{\mathrm{S}}} \frac{1}{\mathrm{C}} \underline{\mathrm{J}} \mathrm{x} \underline{\mathrm{B}}=0
$$

where $N_{s}=\int d^{3} v\left(f_{*_{s}}+h_{s}\right)$ is the number density and $\underline{J}_{\perp}=\Sigma_{s=1, n} Z_{s} e \int d^{3} v \underline{w}\left(f_{*_{s}}+h_{s}\right)$ the perpendicular (to $\underline{B}$ ) component of the current density vector, whereas $\underline{\underline{S}}=\Sigma_{s=1, n} M_{s} \int d^{3} v \underline{v v}\left(f_{*_{s}}+h\right)$ is related to the stress tensor $\underline{\underline{P}}=\Sigma_{s=1, n} M_{s} \int d^{3} v(\underline{v}-\underline{V})$ $(\underline{\mathbf{v}}-\underline{\mathrm{V}})\left(\mathrm{f}_{*_{\mathrm{s}}}+\mathrm{h}_{\mathrm{s}}\right)$, in terms of the relationship:

$$
\underline{\underline{\mathrm{S}}}=\underline{\underline{\mathrm{P}}}+\Sigma_{\mathrm{s}=1, \mathrm{n}} \mathrm{M}_{\mathrm{s}} \int \mathrm{d}^{3} \mathrm{v}(\underline{\mathrm{vV}}+\underline{\mathrm{Vv}}-\underline{\mathrm{VV}})\left(\mathrm{f}_{*_{\mathrm{s}}}+\mathrm{h}_{\mathrm{s}}\right) .
$$

To evaluate to first order the tensor $\underline{\underline{S}}$, it sufficies to take into account the contribution due to the equilibrium distribution $\mathrm{f}_{\mathbf{M}, s}(\underline{\underline{r}}, \underline{\mathbf{y}}, t)$, yielding:

$$
\underline{\underline{S}}=\Sigma_{s=1, n}\left\{M_{s} \bar{N}_{s} \underline{V V}+T_{0, s} \bar{N}_{s}\right\}
$$

To evaluate the current density $\underline{\mathbf{J}}_{\perp}$, instead, one needs to take into account also the contribution of first order to the distribution, yielding from Eqs. (2.83) and (2.84):

$$
\underline{\mathrm{J}} \mathrm{x} \underline{\mathrm{B}}=-\underline{\mathrm{B}} \mathrm{x} \Sigma_{\mathrm{s}=1, \mathrm{n}}\left\{\mathrm{Z}_{\mathrm{s}} \mathrm{e} \overline{\mathrm{N}}_{\mathrm{s}} \mathrm{V}+\int \mathrm{d}^{3} \mathrm{v}(\underline{\mathrm{v}}-\underline{\mathrm{V}}) \tilde{\mathrm{g}}_{\mathrm{D}_{\mathrm{s}}}\right\}
$$

and after evaluating the velocity space integrals: 


$$
\underline{\mathrm{J}}_{\perp}=\mathrm{cRe} \hat{\mathrm{F}}_{\mathrm{s}=1, \mathrm{n}}\left[\frac{\partial}{\partial \Psi}\left(\overline{\mathrm{N}}_{\mathrm{s}} \mathrm{T}_{0, \mathrm{~s}}\right)-\mathrm{M}_{\mathrm{s}} \overline{\mathrm{N}}_{\mathrm{s}}<\omega>2 \mathrm{R} \frac{\partial \mathrm{R}}{\partial \Psi}\right]
$$

where $\mathrm{p}=\Sigma_{\mathrm{s}=1, \mathrm{n}} \mathrm{T}_{\mathrm{o}, \mathrm{s}} \overline{\mathrm{N}}_{\mathrm{s}}$ denotes the scalar pressure. Let us now evaluate $\nabla . \underline{\underline{S}}$ in terms of Eq. (E.5); there results:

$$
\nabla \cdot \underline{\underline{S}}=\nabla \mathrm{p}+\Sigma_{\mathrm{s}=: 1, \mathrm{n}} \mathrm{M}_{\mathbf{s}} \mathbf{N} \underline{\mathrm{V}} \cdot \nabla \underline{\mathrm{V}}
$$

where $\underline{\mathrm{V}} \cdot \nabla \underline{\mathrm{V}}=\omega^{2} \mathrm{R} \hat{e}_{\vartheta} \nabla\left(\mathrm{R} \hat{e}_{\vartheta}\right)=-\omega^{2} \mathrm{R} \hat{e}_{\mathrm{R}}$ and $\hat{\mathrm{e}}_{\mathrm{R}}=\nabla \mathrm{R}$. Letting $\nabla \Psi \frac{\partial \mathrm{R}}{\partial \Psi}+\nabla \chi \frac{\partial \mathrm{R}}{\partial \chi}$ and similarly $\nabla \mathrm{p}=\nabla \Psi \frac{\partial \mathrm{p}}{\partial \Psi}+\nabla \chi \frac{\partial \mathrm{p}}{\partial \chi}$, the poloidal component of $\nabla . \underline{\underline{S}}$ vanishes identically, since:

$$
\frac{\partial \mathrm{p}}{\partial \chi}-\Sigma_{\mathrm{s}=1, \mathrm{n}} \mathrm{M}_{\mathrm{s}} \mathbf{N}_{\mathrm{s}}<\omega>2 \mathrm{R} \frac{\partial \mathrm{R}}{\partial \chi}=0
$$

whereas the radial ( along $\hat{e}_{\Psi}$ ) component reads:

$$
(\nabla \cdot \underline{\underline{S}}) \cdot \hat{e}_{\Psi}=|\nabla \Psi| \Sigma_{s=1, n}\left[\frac{\partial}{\partial \Psi}\left(\mathbb{N}_{s} T_{0, s}\right)-M_{s} \bar{N}_{s}<\omega>2 R \frac{\partial R}{\partial \Psi}\right]
$$

and similarly, since $\operatorname{Re} \hat{\vartheta}_{\vartheta} \underline{B}=\nabla \Psi$ and $\operatorname{Re} \hat{\vartheta}_{\vartheta} \times \underline{B} \cdot \hat{e}_{\Psi}=|\nabla \Psi|$, Eq. (E.8) gives:

$$
\frac{1}{\mathrm{c}} \underline{\mathrm{J}}_{\perp} \times \underline{B} \cdot \hat{\mathrm{e}}_{\Psi}=|\nabla \Psi| \Sigma_{\mathrm{s}=1, \mathrm{n}}\left[\frac{\partial}{\partial \Psi}\left(\mathbb{N}_{\mathrm{s}} \mathrm{T}_{0, \mathrm{~s}}\right)-\mathrm{M}_{\mathrm{s}} \overline{\mathrm{N}}_{\mathrm{s}}<\omega>2 \mathrm{R} \frac{\partial \mathrm{R}}{\partial \Psi}\right] \text {. }
$$

Comparing Eqs. (E.11) and (E.12), one finds that indeed Eq. (E.4) is identically satisfied. Ampere's law [Eq. (4.11)] then gives the equation of magnetostatic equilibrium Eq. (4.10).

Finally, in analogy to Reference [2], to derive the equation advancing in time $I(\Psi, t)$ and the generalized Grad-Shafranov equation yielding $\Psi$ at time $t$, let us project the Ampere law along the directions $\hat{e}_{\chi}$ and $\hat{e}_{\vartheta}$ to obtain:

$$
\begin{aligned}
& \nabla \times \underline{B} \cdot \hat{e}_{\chi}=\frac{4 \pi}{\mathrm{c}} \frac{\mathrm{B}}{\mathrm{B}} \Sigma_{\mathrm{s}=1, \mathrm{n}} \mathrm{Z}_{\mathrm{s}} \mathrm{e} \int \mathrm{d}^{3} \mathrm{vuh}{ }_{1, \mathrm{~s}} \\
& \nabla \mathrm{x} \cdot \hat{\mathrm{e}}_{\vartheta}=4 \pi \mathrm{R} \Sigma_{\mathrm{s}=1, \mathrm{n}}\left[\frac{\partial}{\partial \Psi}\left(\mathrm{N}_{\mathrm{s}} \mathrm{T}{ }_{0, \mathrm{~s}}\right)-\mathrm{M}_{\mathrm{s}} \bar{N}_{\mathrm{s}}<\omega>2 \mathrm{R} \frac{\partial \mathrm{R}}{\partial \Psi}\right]
\end{aligned}
$$

where, since Eq. (2.1) gives $\nabla \times \underline{B}=-R \hat{e}_{\vartheta} \nabla \cdot\left(R^{-2} \nabla \Psi\right)+\frac{1 \partial I}{R \partial \Psi} \nabla \Psi \times \hat{e}_{\vartheta}$ and

$\nabla \Psi \times \hat{e}_{\vartheta}=R \underline{B}_{P}$, there results respectively:

$$
\begin{aligned}
& \frac{\partial \mathrm{I}}{\partial \Psi}=\frac{4 \pi}{\mathrm{cB}} \Sigma_{\mathrm{s}=1, \mathrm{n}} \mathrm{Z}_{\mathrm{s}} \mathrm{e} \int \mathrm{d}^{3} \mathrm{vuh}_{1, \mathrm{~s}} \\
& \nabla \cdot\left(\mathrm{R}^{-2} \nabla \Psi\right)=-4 \pi \Sigma_{\mathrm{s}=1, \mathrm{n}}\left[\frac{\partial}{\partial \Psi}\left(\mathbb{N}_{\mathrm{s}} \mathrm{T}_{0, \mathrm{~s}}\right)-\mathrm{M}_{\mathrm{s}} \mathrm{N}_{\mathrm{s}}\left\langle\omega>2 \mathrm{R} \frac{\partial \mathrm{R}}{\partial \Psi}\right] .\right.
\end{aligned}
$$


Dr. F. Paoloni, Univ. of Wollongong, AUSTRALIA

Prof. M.H. Brennan, Univ. of Sydney, AUSTRALIA

Plasma Research Lab., Australian Nat. Univ., AUSTFALIA

Prof. I.R. Jones, Flinders Univ, AUSTRALIA

Prof. F. Cap, Inst. for Theoretical Physics, AUSTRIA

Prof. M. Heindler, Institut fur Theoretische Physik, AUSTRIA

Prof. M. Goossens, Astronomisch instituut, BELGIUM

Ecole Royaje Militaire, Lab. de Phy. Plasmas, BELGIUM

Commission-European, DG. XII-Fusion Prog., BELGIUM

Prof. R. Bouciqué, Rijksuniversiteit Gent, BELGIUM

Dr. P.H. Sakanaka, Instituto Fisica, BRAZIL

Instituto Nacional Do Pesquisas Espaciais-INPE, BPAZIL

Documents Office, Atomic Energy of Canada Lid., CANADA

Dr. M.P. Bachynski, MPB Technologies, Inc., CANADA

Dr. H.M. Skarsgard, Univ. of Saskatchowan, CANADA

Prof. J. Teichmann, Univ. of Montreal, CANADA

Prof. S.R. Sroenivasan, Univ. of Calgary, CANADA

Prof. T.W. Johnston, INRS-Energie, CANADA

Dr. R. Bolton, Centre canadien de fusion magnétique, CANADA

Dr. C.R. James., Univ. of Alberta, CANADA

Dr. P. Lukác, Komenského Universzita, CZECHO-SLOVAKIA

The Librarian, Culham Laboratory, ENGLAND

Library, R61, Ruthertord Appleton Laboratory, ENGLAND

Mrs. S.A. Hutchinson, JET Library, ENGLAND

Dr. S.C. Sharma, Univ. of South Pacific, FIJI ISLANDS

P. Mahönen, Univ. of Helsinki, FINLAND

Prof. M.N. Bussac, Ecole Polytochnique,. FRANCE

C. Mouttet, Lab. de Physique des Milieux lonisés, fRANCE

J. Radet, CEN/CADARACHE - Bat 506, FRANCE

Prof. E. Economou, Univ. of Crete, GREECE

Ms. C. Rinni, Univ. of loannina, GREECE

Dr. T. Mual, Academy Bibliographic Ser., HONG KONG

Preprint Library, Hungarian Academy of Sci., HUNGARY

Dr. B. DasGupta, Sana Inst. of Nuclear Physics, INDIA

Dr. P. Kaw, Inst. for Plasma Research, INDIA

Dr. P. Rosenau, Israel Inst. of Technology, ISRAEL

Librarian, Intemational Center for Theo Physics, ITALY

Miss C. De Palo, Associazione EURATOM-ENEA, ITALY

Dr. G. Grosso, Istituto di Fisica del Plasma, ITALY

Pro! G. Pnstangni, Istituto Gas lonizzati Dol Cnr, ITALY

Dr. H. Yamato, Toshiba Res \& Devel Conter, JAPAN
Prof. I. Kawakami, Hiroshima Univ., JAPAN

Prof. K. Nishikawa, Hiroshima Univ., JAPAN

Director, Japan Atomic Energy Research Inst., JAPAN

Prof. S. Itoh, Kyushu Univ., JAPAN

Ressarch info. Crr., National Instit. for Fusion Science, JAPAN

Prot. S. Tanaka, Kyoto Univ., JAPAN

Library, Kyoto Univ., JAPAN

Prot. N. Inowe, Univ. of Tokyo, JAPAN

Secretary, Plasma Section, Electrotechnical Lab., JAPAN

S. Mori; Technical Advisor, JAERI, JAPAN

Dr. O. Mitarai, Kumamoto Inst. of Technology, JAPAN

J. Hyeon-Sook, Korea Atomic Energy Research Inst., KOREA

D.I. Choi, The Korea Adv. Inst. of Sci. \& Tech., KOREA

Prof. B.S. Liley, Univ. of Waikato, NEW ZEALAND

Inst of Physics, Chinese Acad Sci PEOPLE'S REP. OF CHINA

Library, Inst. of Plasma Physics, PEOPLE'S REP. OF CHINA

Tsinghua Univ. 'jbrary, PEOPLE'S REPUBLIC OF CHINA

Z. Li, S.W. Inst Physics, PEOPLE'S REPUBLIC OF CHINA

Prof. J.A.C. Cabral, Instituto Superior Tecrico, PORTUGAL

Dr. O. Petrus, AL I CUZA Univ., ROMANIA

Dr. J. de Villiers, Fusion Studies, AEC, S. AFRICA

Prof. M.A. Hellberg, Univ. of Natal, S. AFRICA

Prof. D.E. Kim, Pohang Inst. of Sci. \& Tech., SO. KOREA

Prot. C.I.E.M.A.T, Fusion Division Library, SPAIN

Dr. L Stonflo, Univ. of UMEA, SWEDEN

Library, Royal Inst. of Technology, SWEDEN

Prof. H. Wilheimson, Chaimers Univ. of Tech., SWEDEN

Centre Phys. Des Plasmas, Ecole Polytech, SWITZERLAND

Bibliothook, Inst. Voor Plasma-Fysica, THE NETHERLANDS

Asst. Prof. Dr. S. Cakir, Middle East Tech. Univ., TURKEY

Dr. V.A. Glukhikh,Sci. Tes. Inst. Eloctrophys.I Apparatus, USSR

Dr. D.D. Ayutov, Siberian Branch of Academy of Sci., USSR

Dr. G.A. Elisoev, I.V. Kurchatov Inst. USSA

Librarian. The Ukr.SSR Academy of Sciences, USSR

Dr. L.M. Kovrizhnykh, Inst. of General Physics, USSR

Kemforschungsanlage GmbH, Zentralbibliothek, W. GERMANY

Bibliothek, Inst. Für Plasmaforschung, W. GERMANY

Prof. K. Schinder, Ruhr-Universitát Bochum, W. GERMANY

Dr. F. Wagner, (ASDEX), Max-Planck-Institut, W. GERMANY

Librarian, Max-Planck-Institut, W. GERMANY

Prot. R.K. Janev, Inst. of Physics, YUGOSLAVIA 

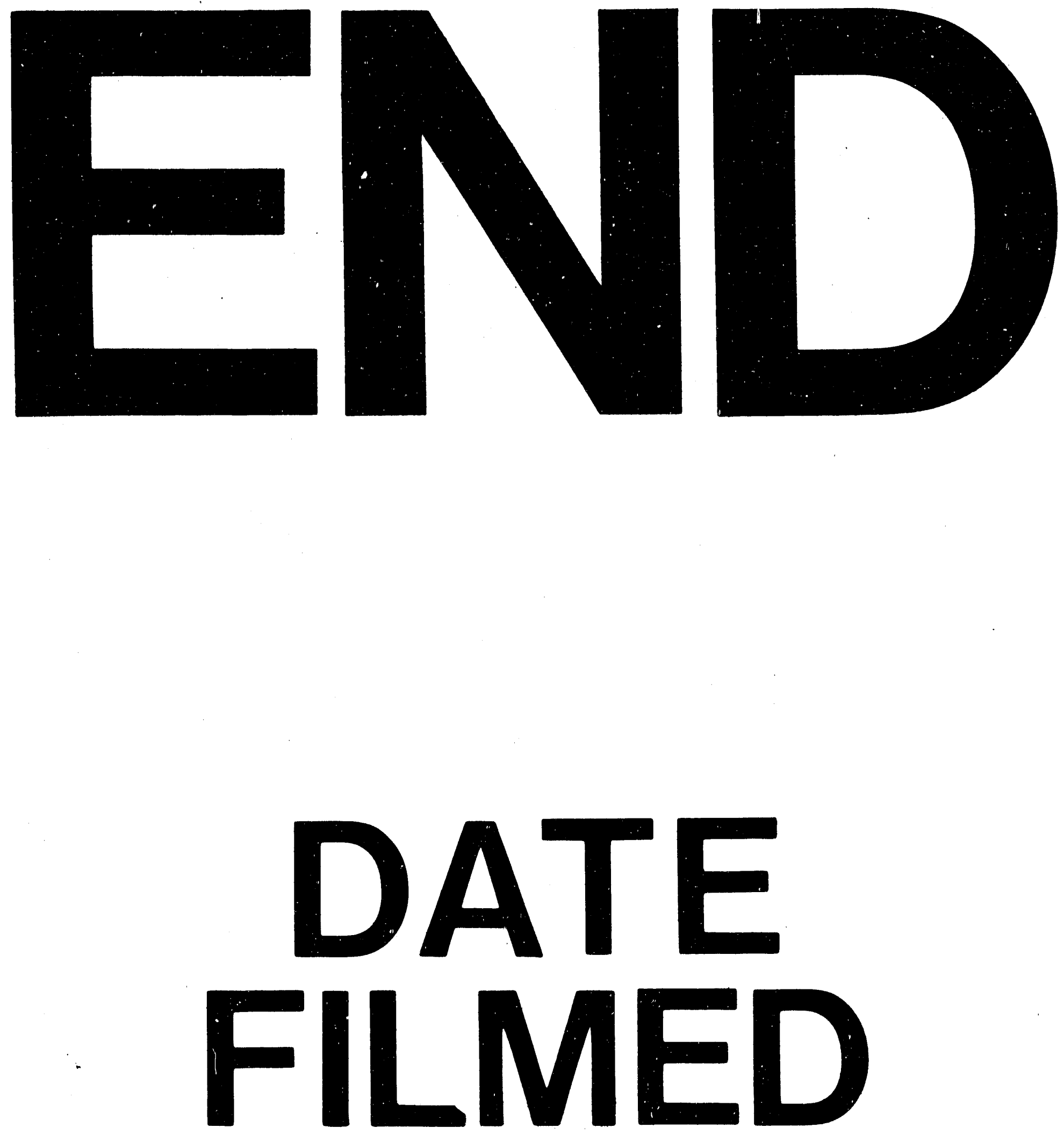

1

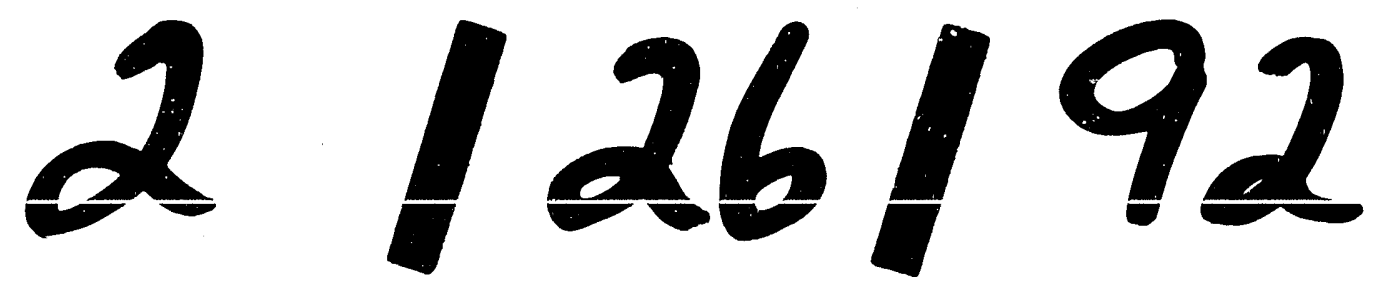




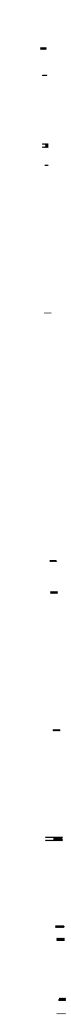

